\title{
Electromagnetic fluid drift turbulence in static ergodic magnetic fields
}

D. Reiser, and B. Scott

Citation: Physics of Plasmas 12, 122308 (2005); doi: 10.1063/1.2141928

View online: https://doi.org/10.1063/1.2141928

View Table of Contents: http://aip.scitation.org/toc/php/12/12

Published by the American Institute of Physics

\section{Articles you may be interested in}

On collisional diffusion in a stochastic magnetic field

Physics of Plasmas 20, 082507 (2013); 10.1063/1.4818145

Collisionality and magnetic geometry effects on tokamak edge turbulent transport. I. A two-region model with application to blobs

Physics of Plasmas 13, 112502 (2006); 10.1063/1.2364858

Predicting core and edge transport barriers in tokamaks using the GLF23 drift-wave transport model

Physics of Plasmas 12, 052503 (2005); 10.1063/1.1886826

Collisionality and magnetic geometry effects on tokamak edge turbulent transport. II. Many-blob turbulence in the two-region model

Physics of Plasmas 14, 102307 (2007); 10.1063/1.2780137

Bifurcated states of a rotating tokamak plasma in the presence of a static error-field

Physics of Plasmas 5, 3325 (1998); 10.1063/1.873000

Impact of resonant magnetic perturbations on nonlinearly driven modes in drift-wave turbulence Physics of Plasmas 19, 055903 (2012); 10.1063/1.3694675

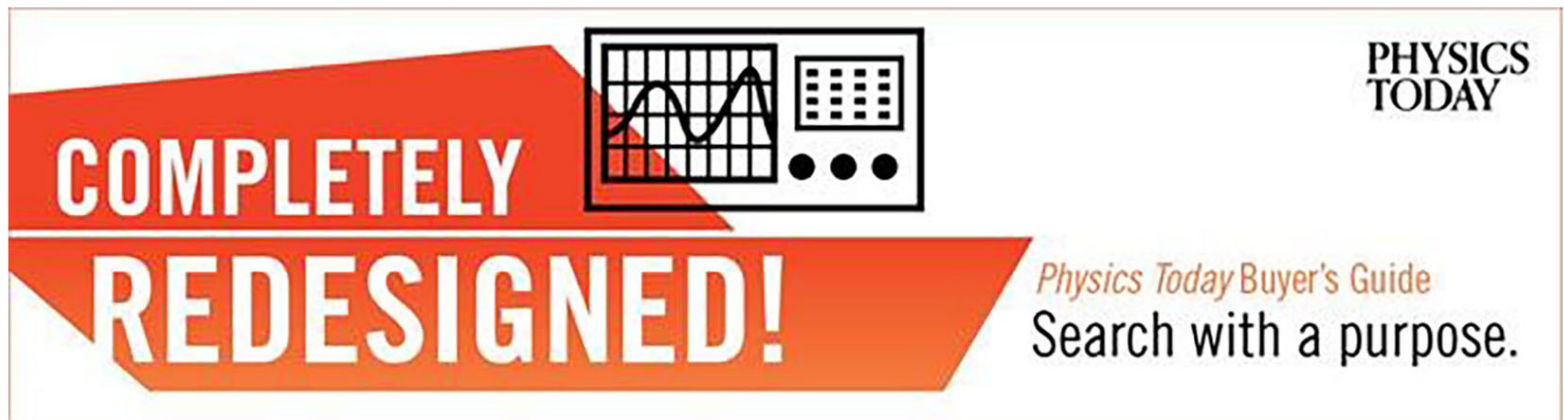




\title{
Electromagnetic fluid drift turbulence in static ergodic magnetic fields
}

\author{
D. Reiser \\ Institut für Plasmaphysik, Forschungszentrum Jülich GmbH, EURATOM Association, \\ Trilateral Euregio Cluster, D-52425 Jülich, Germany \\ B. Scott \\ Max-Planck-Institut für Plasmaphysik, EURATOM Association, D-85748 Garching, Germany
}

(Received 6 July 2005; accepted 3 November 2005; published online 20 December 2005)

\begin{abstract}
Numerical simulations of three-dimensional nonlinear electromagnetic fluid drift turbulence in a tokamak plasma with externally applied stochastic magnetic-field perturbations are presented. The contributions to the radial particle transport due to nonlinearities arising from $E \times B$ advection and magnetic flutter are investigated for perturbation fields of varying strengths in the cases of low and high collisionalities. The perturbation strength is varied to study the physics for Chirikov parameters above 1. In all the cases considered a significant increase of $E \times B$ transport is found. A static contribution in the density and velocity perturbations contributes significantly to the total radial $E \times B$ transport. For low collisionality, the external perturbation leads to enhanced density and velocity fluctuations over a broad range in the toroidal wave-number spectrum, resulting in an enhanced turbulent flux. For high collisionality, the density fluctuations stay roughly the same and the velocity fluctuations are increased in an intermediate range of the toroidal wave number spectrum, separated from the maximum of the density fluctuations, thus leaving the turbulent flux almost unchanged. () 2005 American Institute of Physics. [DOI: 10.1063/1.2141928]
\end{abstract}

\section{INTRODUCTION}

Stochastic magnetic fields and their effect on particle transport across magnetic surfaces are inherently of interest in plasma physics, in particular, fusion-oriented research. ${ }^{1-8}$ On one hand certain regions around magnetic islands in stellarators and near the separatrix in tokamak divertors always exhibit some stochasticity. On the other hand a well-directed induced magnetic-field perturbation external to the plasma volume might offer the possibility to control certain kinds of instabilities and turbulence responsible for anomalous transport degrading the confinement properties. Experimental setups to study the impact of such ergodization fields have been realized, e.g., at the tokamak devices Tore-Supra ${ }^{9-11}$ and Tokamak Experiment for Technology Oriented Research with Dynamic Ergodic Divertor ${ }^{12-14}$ (TEXTOR-DED). Experimental results at Tore-Supra have been interpreted as indicating a strongly modified turbulent behavior in the edge plasma region leading to a decrease of density fluctuations while the turbulent cross field diffusivity is unaffected. Moreover the ergodized magnetic field offers an additional channel of particle and heat transport to the walls which can be utilized to cool the plasma locally and pull out energy and particles in a controlled way. To study the impact of stochastic magnetic fields, simplified analytical fluid models have been employed to consider the enhanced radial transport. ${ }^{15-17}$ These models estimate the additional transport in radial direction along the perturbed magnetic-field lines assuming that the underlying turbulent transport is unchanged. They have shown that for a certain degree of stochastization the mass flow due to parallel flow transport in radial direction can be much stronger than the seed diffusion due to turbulence. An attempt to investigate the modifications in the electrostatic turbulence has been done by a three-dimensional numerical modeling of resistive magnetohydrodynamic (MHD) ballooning turbulence for Tore-Supra scenarios using the RBM3D code. ${ }^{18,19}$ This showed that the density fluctuations in resistive MHD ballooning turbulence are decreased but the turbulent $E \times B$ transport is unaffected by a static ergodic magnetic field. The studies were, however, limited to the single-fluid MHD approximations, which assume, in particular, that pressure forces on the electrons cannot compete with the electric field. The self-correcting back reaction of the electrons to force countercurrents is therefore neglected. The self-consistent magnetic fluctuations produced by pressure-gradient-driven turbulence are also neglected (i.e., by neglecting the attendant drift wave physics). It has to be noted that the MHD approximation in the RBM3D simulations has been removed partly by the introduction of a dissipative term in the density equation, reflecting a piece of the driftAlfvén dynamics, i.e., the coupling of density and current.

In this work a two-fluid model which does not make the MHD assumptions is applied to this problem. We employ the DALF3 code ${ }^{20,21}$ which uses the four-field version of the dissipative drift-Alfvén model ${ }^{22}$ to compute the nonlinear electromagnetic fluid drift turbulence in the presence of a static magnetic ergodization field. The self-consistent magnetic flutter effects associated with the interaction of the drift wave dynamics in the $E \times B$ turbulence and the induced magnetic disturbances are thereby taken into proper account. The twofield resistive MHD ballooning model mentioned above is a wholly contained subset of this four-field model. Thus our approach allows us to study the turbulence characteristics from a more general point of view (a more detailed discussion of this point is given in Sec. V).

We describe computations made for realistic edge plasma parameters and perturbation fields of TEXTOR-DED. 
The parameters are chosen for two scenarios with low and high collisionalities, respectively (but keeping the plasma $\beta$ constant), to study the difference of drift wave driven and strong ballooning turbulence in stochastic fields. In all the cases considered, we find a significant increase in the density and potential perturbations and in the $E \times B$ transport, when moving from unperturbed magnetic fields to stochastic magnetic fields (Chirikov parameter $\sigma>1$ ). The strong ballooning scenarios are less affected than the drift wave driven. An analysis of the energy flow in the model system shows that the character of the turbulence is preserved in static stochastic fields. By time averaging the numerical solutions of the model equations the stationary solutions are approximated and it is found that strong resonant effects can be attributed to (quasi-) static contributions of the perturbations in the density, electrostatic potential, magnetic potential, and parallel ion velocity at low toroidal mode numbers $(n<40)$. Considering the fluctuating parts a very different behavior of the low and high collisional cases appears. In particular, the density fluctuations and the corresponding turbulent transport show completely different tendencies with increasing magnetic perturbations in that they are almost unchanged in a high collisional plasma, but are increased strongly for low collisionality.

The structure of the paper is as follows: in Sec. II basic features of the DALF3 code, namely, the underlying model (Sec. II A) and its treatment of the magnetic geometry (Sec. II B), are recalled. The implementation of externally induced stochastic magnetic fields is addressed in Sec. II C. The specific parameters and the simulation setup for numerical studies on TEXTOR-DED are elucidated in Sec. III. In Sec. IV results of the numerical simulations are presented, which are summarized and discussed in Sec. V. Finally a brief conclusion is given in Sec. VI.

\section{TURBULENCE MODEL AND NUMERICAL APPROACH}

\section{A. Four-field fluid drift model}

We employ the DALF3 $\operatorname{code}^{20,21}$ to perform the turbulence simulations. This code uses the most basic model containing the principal interactions of dissipative drift wave physics in a general closed magnetic-flux tube geometry, represented by a four-field model ${ }^{22}$ describing the nonlinear evolution of small scale dynamics of the electrostatic potential $\widetilde{\phi}$, the density $\widetilde{n}$, the parallel magnetic potential $\widetilde{A}_{\|}$, and the parallel ion velocity $\widetilde{u}_{\|}$. The equations are derived from the general two-fluid equations using drift ordering ${ }^{23-25}$ and appear as

$$
\frac{d \tilde{n}}{d t}=-\widetilde{\mathbf{v}}_{E} \cdot \nabla n+\frac{1}{e} B \nabla_{\|} \frac{\widetilde{J}_{\|}}{B}-n B \nabla_{\|} \frac{\widetilde{u}_{\|}}{B}+n \mathcal{K}(\widetilde{\phi})-\frac{T_{e}}{e} \mathcal{K}(\widetilde{n}),
$$

$$
\frac{\partial \widetilde{A}_{\|}}{\partial t}+\frac{m_{e}}{n e^{2}} \frac{d \widetilde{J}_{\|}}{d t}=\frac{T_{e}}{n e} \nabla_{\|}(n+\widetilde{n})-\nabla_{\|} \widetilde{\phi}-\eta_{\|} \widetilde{J}_{\|},
$$

$$
\begin{aligned}
& n M_{i} \frac{d \widetilde{u}_{\|}}{d t}=-T_{e} \nabla_{\|}(n+\widetilde{n}), \\
& \frac{n M_{i}}{B^{2}} \frac{d}{d t} \nabla_{\perp}^{2} \widetilde{\phi}=B \nabla_{\|} \frac{\widetilde{J}_{\|}}{B}-T_{e} \mathcal{K}(\widetilde{n}) .
\end{aligned}
$$

These are the equation of continuity, Ohm's law, the total momentum balance, and the quasineutrality condition $\boldsymbol{\nabla} \cdot \tilde{\mathbf{J}}$ $=0$, respectively. Here $\eta_{\|}$is the classical parallel resistivity, ${ }^{26}$ $m_{e}$ and $M_{i}$ are the electron and ion masses, $n$ is the equilibrium density $\left(n=n_{e}=n_{i}\right)$, and $T_{e}$ is the electron temperature. The parallel magnetic potential and current density are related by Ampère's law $\mu_{0} \widetilde{J}_{\|}=-\nabla_{\perp}^{2} \widetilde{A}_{\|}$. A remark on the notation might be necessary at this point. The tilde in the notation is used to label the scalar quantities as perturbations of a given axisymmetric background equilibrium plasma (nontilded quantities), which is characterized by a constant radial background density gradient. It should not be confused with fluctuations because stationary and axisymmetric solutions are, in principle, possible and might be assigned to a modified background plasma. The distinction between (quasi-) stationary solutions and time-dependent fluctuations will be of particular importance in the discussion of the simulation results in Sec. IV D. The convention used in the following is that we call the tilded quantities the "perturbations" and their time-dependent pieces are denoted by "fluctuations." The operators appearing in the model equations are defined as follows. The nonlinear time derivative and parallel gradient

$$
\frac{d}{d t}=\frac{\partial}{\partial t}+\widetilde{\mathbf{v}}_{E} \cdot \boldsymbol{\nabla}, \quad \boldsymbol{\nabla}_{\|}=\frac{\mathbf{B}+\widetilde{\mathbf{B}}_{\perp}}{B} \cdot \boldsymbol{\nabla}
$$

include advection by the $E \times B$ velocity $\widetilde{\mathbf{v}}_{E}$ and the magnetic flutter effect due to shear-Alfvén-type magnetic disturbances $\widetilde{\mathbf{B}}_{\perp}=\widetilde{\mathbf{B}}_{\text {int }}+\widetilde{\mathbf{B}}_{\text {ext }}$, given by

$$
\widetilde{\mathbf{v}}_{E}=\frac{\mathbf{B} \times \nabla \tilde{\phi}}{B^{2}}
$$

and

$$
\widetilde{\mathbf{B}}_{\text {int }}=-\frac{\mathbf{B} \times \nabla \widetilde{A}_{\|}}{B}, \quad \widetilde{\mathbf{B}}_{\mathrm{ext}}=-\frac{\mathbf{B} \times \boldsymbol{\nabla} \widetilde{A}_{D}}{B},
$$

where $\widetilde{A}_{\|}$is the self-consistent parallel magnetic potential and $\tilde{A}_{D}$ is an externally applied static perturbation field with zero current. Correspondingly we call $\widetilde{\mathbf{B}}_{\text {int }}$ the intrinsic magnetic flutter and $\widetilde{\mathbf{B}}_{\text {ext }}$ the externally induced magnetic flutter. We note that the form of the applied perturbation field reflects a scenario with external coils rather than an inductive drive which would involve local currents. The curvature operator $\mathcal{K}$ denotes the quasistatic compression of $E \times B$ and diamagnetic flows in a toroidal magnetic field. The perpendicular Laplacian $\nabla_{\perp}^{2}$ describes the derivatives in the local drift plane, perpendicular to the magnetic field, relating field quantities (electrostatic and parallel magnetic potentials) to the plasma-dependent variables (vorticity and parallel current). The implementation of these operators depends on the coordinate system and is given below. 
Drift ordering refers to the neglect of parallel compared to perpendicular derivatives (i.e., everywhere except in $\boldsymbol{\nabla}_{\|}$) and the neglect of all nonlinearities except for the action of $E \times B$ advection or magnetic flutter. The background is a set of constant parameters except where these two operators act upon $n$. The background gradient $\nabla n$ is replaced by $\left(-n / L_{\perp}\right) \nabla x$, defining the radial coordinate $x$ and the profile scale length $L_{\perp}$. Apart from the neglect of dissipation and extra instabilities due to fluctuations in the temperature, this model forms a quite general set including (linear) physics such as sound waves, drift Alfvén waves, and drift-resistive ballooning modes. The inclusion of the magnetic flutter and the induction term in Ohm's law makes it an electromagnetic model. A more detailed discussion of the basic physics described by the model can be found in Refs. 22 and 27 and references therein.

Application of this model or similar sets in studies on electromagnetic turbulence is reported in Refs. 20 and 2730. In studies on resistive MHD ballooning turbulence in Tore-Supra (RBM3D code) a two-field electrostatic MHD model was used, i.e., sound waves and Alfvénic dynamics besides parallel electron self-consistency have been neglected, but externally induced perturbations in the magnetic field have been retained. ${ }^{18,19}$ This model is entirely contained as a reduced subset in our model equations and can be recovered by retaining only the first two terms in Eq. (1) and the last two terms in Eq. (2), leaving Eq. (4) unmodified but neglecting Eq. (3) entirely [a later version also keeps the $\mathcal{K}(\phi)$ term in Eq. (1), as it should to conserve energy properly]. As mentioned in the Introduction the third term in Eq. (1) is taken into account partly to keep a certain collisional damping of the density perturbations (see the Appendix and Sec. V).

\section{B. Magnetic geometry and differential operators}

The other two coordinates besides the radial one describe the geometry within the magnetic surface. These are defined by aligning to the magnetic field, such that only one contravariant component of the undisturbed magnetic field is nonvanishing. ${ }^{31,32}$ Global consistency in the boundary conditions is maintained, ${ }^{32}$ and a shifted metric treatment is used, defining a separate global coordinate system on each perpendicular drift plane, taking the shifts in the drift angle coordinate $(y)$ when evaluating undisturbed parallel derivatives (in the $s$ coordinate), thereby allowing equally precise representation of sheared slab and interchange/ballooning physics. ${ }^{20}$

Our studies are confined to a radially thin region of the (ergodized) edge plasma region which lies inside the last closed surface of the underlying unperturbed equilibrium magnetic field of an axisymmetric tokamak. The equilibrium magnetic field is prescribed by the contravariant components in toroidal coordinates $\{r, \theta, \varphi\}$ as

$$
B^{r}=0, \quad B^{\theta}=\frac{B_{0}}{q R}, \quad B^{\varphi}=\frac{B_{0}}{R},
$$

where $R=R_{0}+r \cos \theta$ with $R_{0}$ and $r$ the major and minor radii, respectively. Here $\theta$ is the poloidal angle and $\varphi$ is the toroidal angle. The magnetic-field strength at the magnetic axis is denoted by $B_{0}$ and $q$ is the pitch parameter (safety factor). We use the field-aligned, shifted metric coordinates, ${ }^{20}$ given by

$$
x=r-r_{0}, \quad y_{k}=\frac{r_{0}}{q_{0}}\left[q\left(\theta-s_{k}\right)-\varphi\right], \quad s=\theta,
$$

where $s_{k}$ is an arbitrary global constant, $r_{0}$ denotes a reference radius, and $q_{0}=q\left(r_{0}\right)$, with $q$ expanded about $x=0$ as $q=q_{0}\left(1+\hat{s} x / r_{0}\right)$. The subscript $k$ refers to the particular fieldaligned coordinate system referenced at $s=s_{k}$, such that the off-diagonal element of the perpendicular metric vanishes there. We use the simplest model of circular flux surfaces with all finite aspect ratio effects neglected except for the existence of the magnetic curvature. The metric coefficients are

$$
g^{x x}=1, \quad g_{k}^{x y}=\hat{s}\left(s-s_{k}\right), \quad g_{k}^{y y}=1+\hat{s}^{2}\left(s-s_{k}\right)^{2},
$$

with a unit Jacobian.

The differential operators of Eqs. (1)-(4) are given by a Poisson bracket structure for the $E \times B$ advection (for any scalar $f$ ),

$$
\widetilde{\mathbf{v}}_{E} \cdot \nabla f=\frac{1}{B_{0}}\left(\frac{\partial \widetilde{\phi}}{\partial x} \frac{\partial f}{\partial y_{k}}-\frac{\partial \widetilde{\phi}}{\partial y_{k}} \frac{\partial f}{\partial x}\right),
$$

a Poisson bracket structure for the magnetic flutter,

$$
\nabla_{\|} f=\frac{1}{q_{0} R_{0}} \frac{\partial f}{\partial s}-\frac{1}{B_{0}}\left(\frac{\partial \tilde{A}_{\|}^{*}}{\partial x} \frac{\partial f}{\partial y_{k}}-\frac{\partial \tilde{A}_{\|}^{*}}{\partial y_{k}} \frac{\partial f}{\partial x}\right)
$$

where $\widetilde{A}_{\|}^{*}=\widetilde{A}_{\|}+\widetilde{A}_{D}$ including both self-consistent and externally induced contributions, the perpendicular Laplacian,

$$
\nabla_{\perp}^{2} f=g^{x x} \frac{\partial^{2} f}{\partial x^{2}}+2 g_{k}^{x y} \frac{\partial^{2} f}{\partial x \partial y_{k}}+g_{k}^{y y} \frac{\partial^{2} f}{\partial y_{k}^{2}},
$$

and the curvature operator,

$$
\mathcal{K}(f)=\frac{2}{B_{0} R_{0}}\left[\sin \theta \frac{\partial f}{\partial x}+\left(\cos \theta+g_{k}^{x y} \sin \theta\right) \frac{\partial f}{\partial y_{k}}\right],
$$

representing the toroidal magnetic divergences.

With the establishment of these definitions the subscript $k$ may be understood within the definition of the drift angle coordinate $(y)$ on each drift plane and it is dropped in the sections below for clarity (note that the partial derivative with respect to any of the $y_{k}$ is the same). Boundary conditions in the two angle coordinates $(y, s)$ follow global consistency of toroidally topological, closed magnetic-flux surfaces, ${ }^{32}$ implemented following the shifted metric procedure. ${ }^{20}$ Further details can be found in Refs. 20 and 32.

\section{Treatment of externally induced magnetic-field perturbations}

In this work we focus on scenarios where external currents (flowing in coils outside the plasma volume) induce a static perturbation in the magnetic field. This reflects the technical realization of ergodization experiments in Tore-Supra $^{9-11}$ or TEXTOR-DED. ${ }^{12-14}$ For this purpose we consider the perturbations in the vector potential to consist of two parts. One is the self-consistent intrinsic plasma re- 
sponse $\widetilde{A}_{\|}$arising from the parallel current as forced by gradients in the electrostatic potential and electron pressure. The other is the constant contribution $\widetilde{A}_{D}$ externally imposed by coil currents, so that $\nabla_{\perp}^{2} \tilde{A}_{D}=0$ within the computational domain. Both pieces appear in the Poisson bracket structure for the magnetic flutter [Eq. (12)]. The extension to dynamic perturbation fields is simply done by introducing $\partial \widetilde{A}_{D} / \partial t$ into Ohm's law as an additional forcing term, but this is left to future work.

For TEXTOR-DED scenarios the externally induced magnetic field can be described by the approximate vector potential, ${ }^{12}$ in terms of the original toroidal coordinates $\{r, \theta, \varphi\}$,

$$
\widetilde{A}_{D}^{\left(n_{0}\right)}=-\sum_{m=-\infty}^{\infty}(-1)^{m+3 n_{0}} g_{m, n_{0}}(r) \cos \left(m \theta-n_{0} \varphi\right),
$$

where

$$
g_{m, n_{0}}(r)=\frac{B_{D} r_{c}}{m}\left(\frac{r}{r_{c}}\right)^{|m|} \frac{\sin \left[\left(m-3 n_{0}\right) \theta_{c}\right]}{\left(m-3 n_{0}\right) \pi}
$$

and $r_{c}=0.5325 \mathrm{~m}$ is the radial position of the perturbation coils, $\theta_{c}=2 \pi / 10$ is half of the poloidal extension of the coil system, and the magnetic field $B_{D}=4 \mu_{0} I_{D} / \theta_{c} r_{c}$ is given as a function of the external current $I_{D}$ in the divertor coils. The reference toroidal mode number is $n_{0}=4$ for the $12 / 4$ mode operation, determined by the coil arrangement in the experiment. The dominant mode is the one with $m=3 n_{0}$, which is resonant at the $q=3$ surface located at $x=0$, i.e., in the center of the computational domain in the radial direction. According to the radially local model used in this work the radial extent of the computational domain is limited to a width of $L_{\perp}$ (background gradient length) and this limits the number of resonances of the perturbation field which can be taken into account. Thus the perturbation field (15) is approximated by a three-mode perturbation using the basic mode numbers $11 / 4,12 / 4$, and $13 / 4$. For the computations $\widetilde{A}_{D}$ must be transformed to the $\{x, y, s\}$ coordinate system, wherein it is important to note that unlike the basic geometry itself, this field perturbation is not axisymmetric. The expression of $\widetilde{A}_{D}^{\left(n_{0}\right)}$ in the shifted metric [Eqs. (9) and (10)] is given by

$$
\widetilde{A}_{D}^{\left(n_{0}\right)}=-\sum_{m_{*}=-\infty}^{\infty}(-1)^{m_{*}} g_{m_{*}, n_{0}}^{*}(x) \cos \left(m_{*} s_{k}+n_{0} K_{y} y_{k}\right),
$$

where

$$
g_{m_{*}, n_{0}}^{*}(r)=\frac{B_{D} r_{c}}{m_{*}+n_{0} q_{0}} e^{n_{0} K_{y}\left(x-x_{c}\right)} \frac{\sin \left(m_{*} \theta_{c}\right)}{m_{*} \pi},
$$

with $x_{c}=r_{c}-r_{0}, m_{*}=m-n_{*} q_{0}$, and $K_{y}=q_{0} / r_{0}$.

Note that our approach is to some extent a cylindrical approximation, and that the exponential radial dependence is necessary to fulfil $\nabla_{\perp}^{2} \widetilde{A}_{D}^{\left(n_{0}\right)}=0$, according to the definition of the Laplacian in Eq. (13).

The use of Eqs. (15)-(18) needs some clarification because it is an approximation for the vacuum field induced by external coils. The question arises if this is the correct per- turbation field to take into account, due to the modification of the perturbation by the plasma response. Those modifications are studied, e.g., in Refs. 12 and 13, and they appear as a change in the amplitudes of the perturbation spectrum. But also our model equations describe partly the intrinsic (perpendicular) magnetic-field response and it would confuse matters to take into account the plasma response twice. In this work we do not attempt to resolve this question, but we consider the model field described by Eqs. (15)-(18) as a prototypical scenario to study the basic effects of overlapping islands of realistic structure, even if the detailed spectrum is approximative.

To estimate the level stochasticity corresponding to the model field described by Eq. (15) with $m=11,12,13$, we consider the island width of the dominant modes as given in Ref. 33,

$$
\delta_{m, n_{0}}=2 \sqrt{\frac{q R_{0}}{\hat{s} B_{0}} g_{m, n_{0}}\left(r_{m, n_{0}}\right)},
$$

where $q$ and $\hat{s}$ are the safety factor and the magnetic shear at the resonant surface located at $r=r_{m, n_{0}}$. The level of stochasticity can be estimated by the Chirikov parameter as a measure for the overlap of the dominant mode island and the neighboring islands,

$$
\sigma_{+}=\frac{\delta_{13,4}+\delta_{12,4}}{\left|r_{13,4}-r_{12,4}\right|}, \quad \sigma_{-}=\frac{\delta_{11,4}+\delta_{12,4}}{\left|r_{11,4}-r_{12,4}\right|} .
$$

Using the parameters $q_{0}=3, r_{0}=r\left(q_{0}\right)=0.45 \mathrm{~m}$, and $\hat{s}=2$ and a linear profile $q=q_{0}\left[1+\hat{s}\left(r-r_{0}\right) / r_{0}\right]$, one obtains for the Chirikov parameters in the $12 / 4$ perturbation $\sigma_{-}=0.83 I_{D}^{1 / 2}$ and $\sigma_{+}=1.25 I_{D}^{1 / 2}$ (with $I_{D}$ in units of kiloamperes). Thus the onset of stochasticity for the model field described by Eq. (15), and characterized by $\sigma=\max \left(\sigma_{-}, \sigma_{+}\right) \geqslant 1$, can be expected in the vicinity of a perturbation current of about $I_{D}$ $=1 \mathrm{kA}$. This is also illustrated by the Poincaré plots in Fig. 1 where field lines have been started inside our computational domain $(2.6 \leqslant q \leqslant 3.4)$ and have been followed for 300 toroidal turns. It is evident that for $I_{D}=1 \mathrm{kA}$ the overlap between the dominant island at $q=3$ and the neighboring islands becomes significant. Besides the Chirikov parameter another important dimensionless quantity to describe the magnetic perturbation field is the ratio $L_{c} / L_{K}$ between the connection length $L_{c}$, i.e., the length of a field line connecting different points of the radial boundaries, and the Kolmogorov length $L_{K}$, an $e$-folding length quantifying the divergence of neighboring field lines due to the magnetic perturbation field. The Kolmogorov length is estimated by $L_{K}=\sigma^{-4 / 3} q_{0} R_{0} \approx 2.5-4 \mathrm{~m}$. The connection length $L_{c}$ is computed numerically for different starting points in our computational domain and the ratio $L_{c} / L_{K}$ for the case with $I_{D}$ $=2 \mathrm{kA}$ is shown in Fig. 2. It can be seen that for the most part of the computational domain the connection length $L_{c}$ is much longer than the Kolmogorov length $L_{K}$; thus the radial transport along the field lines is dominated by stochasticity and not by a direct shortcut between points on the boundaries, and thus by boundary conditions. In particular, the region of island overlap is characterized by $L_{c} / L_{K} \gg 1$ and only 

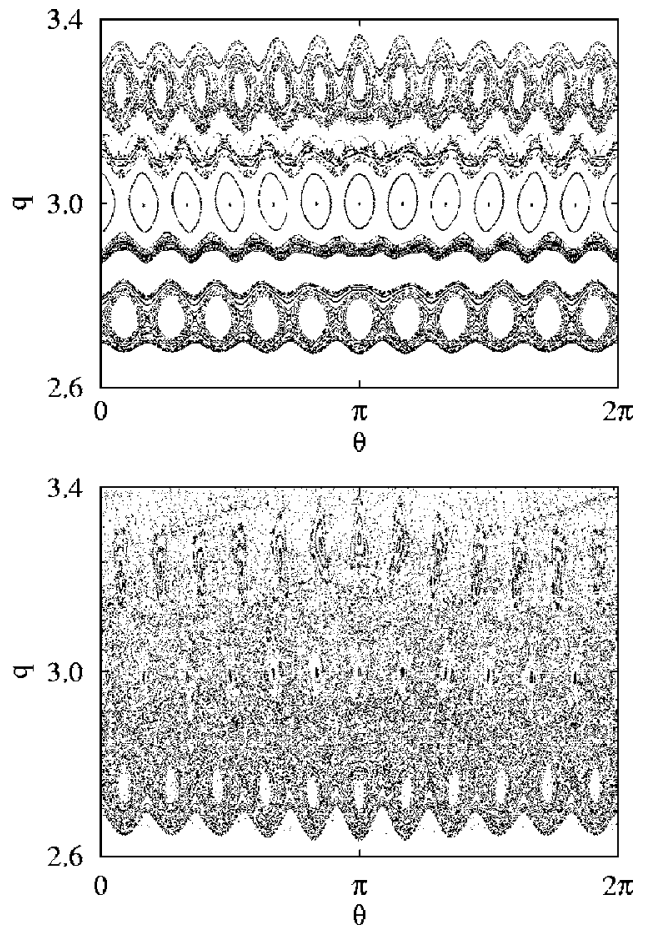

FIG. 1. Poincaré plots for the $12 / 4$ perturbation field for $I_{D}=0.3 \mathrm{kA}$ $\left(\sigma<1\right.$, top) and $I_{D}=1.0 \mathrm{kA}(\sigma>1$, bottom). The field lines have been started inside the computational domain where $2.6 \leqslant q \leqslant 3.4$ and have been followed along 300 toroidal turns.

in a small region close to the radial edges that we find $L_{c} / L_{K} \approx 2$.

\section{SETUP OF NUMERICAL COMPUTATIONS}

In the turbulence simulations we consider dynamics in a thin radial range (motivating the use of radially local geometry), but due to the long-wavelength character of $\widetilde{A}_{D}$ we carry the entire flux surface (in terms of Refs. 20 and 32 the toroidal truncation number is $k_{0}=1$ ). The $x y$ domain is extremely anisotropic, since the perpendicular distance within the flux surface between two successive cuts by the same field line, $L_{y}=2 \pi r_{0} / q_{0}$, is much larger than any reasonable radial domain size. The radial domain is contained within a single profile scale length $L_{\perp}$, as required by the use of para-

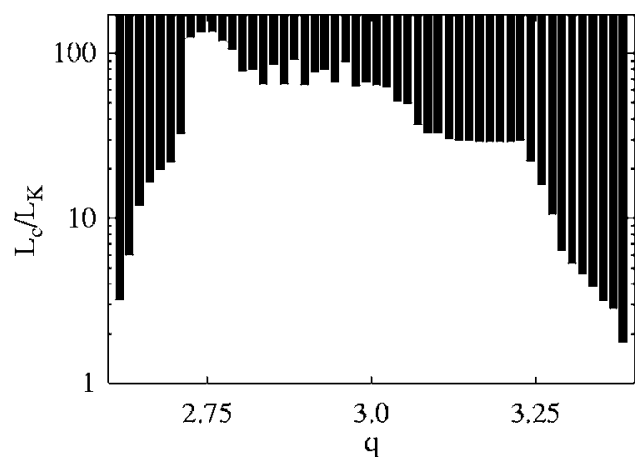

FIG. 2. Plot of the ratio $L_{c} / L_{K}$ for $I_{D}=2 \mathrm{kA}$. The field lines have been started inside the computational domain where $2.6 \leqslant q \leqslant 3.4$ and have been followed along 20 toroidal turns. metrically homogeneous equations (for further commentary on the setup of edge turbulence computations see Ref. 34). The resolution is set equal in $x$ and $y$, so the corresponding grid node counts $N_{x}$ and $N_{y}$ are disparate by more than a factor of 10. The $s$ domain is given by the global consistency constraint. The spatial domain is given by

$$
\begin{aligned}
& -\frac{N_{x}}{N_{y}} \frac{\pi r_{0}}{q_{0}} \leqslant x \leqslant \frac{N_{x}}{N_{y}} \frac{\pi r_{0}}{q_{0}}, \\
& -\frac{\pi r_{0}}{q_{0}} \leqslant y \leqslant \frac{\pi r_{0}}{q_{0}}, \\
& -\pi \leqslant s \leqslant \pi .
\end{aligned}
$$

Given the pitch parameter expansion $q=q_{0}\left(1+\hat{s} x / r_{0}\right)$ the helicity of the magnetic field takes the values

$$
q_{0}-\frac{N_{x}}{N_{y}} \hat{s} \pi \leqslant q \leqslant q_{0}+\frac{N_{x}}{N_{y}} \hat{s} \pi .
$$

The boundary conditions for the dependent variables in the computations are Dirichlet for $x$ (vanishing at the boundaries), fixing the profile at both boundaries but allowing fully self-consistent evolution in the interior, periodic for $y$, following the toroidal periodicity, and pseudoperiodic in $s$,

$$
f(x, y, s+2 \pi)=f(x, y-2 \pi \hat{s} x, s),
$$

following global consistency. ${ }^{32}$ When the shifted metric is implemented, Eq. (23) becomes ${ }^{20}$

$$
f\left(x, y_{k+N}, s_{k+N}\right)=f\left(x, y_{k}, s_{k}\right),
$$

where $N$ is the number of nodes in the $s$ direction and $s_{k+N}$ $=s_{k}+2 \pi$. The Dirichlet boundary conditions in the $x$ direction for the hyperbolic model equations have the consequence that (linear) perturbation waves moving towards the radial boundary are allowed to pass the boundary without any effect on them. On the other hand, no perturbation can enter the computational domain, thereby neglecting effects from the outside like, e.g., the impact of a conducting wall intersected by magnetic-field lines leaving the domain. To extend the model to such scenarios, one would have to include scrape-off-layer and sheath physics, which is beyond the scope of the present work. The boundary effects mentioned in Sec. II C due to our use of a perturbation field ranging from one radial edge to the other are expected to be small.

For the simulation of edge turbulence we use parameters representative of typical experimental scenarios in the TEXTOR-DED. A low collisional case is set up by $n=2$ $\times 10^{19} \mathrm{~m}^{-3}, T=100 \mathrm{eV}, r_{0}=0.45 \mathrm{~m}, R_{0}=1.75 \mathrm{~m}, q_{0}=3, \hat{s}$ $=2, L_{\perp}=0.03 \mathrm{~m}, B_{0}=2 \mathrm{~T}$, and $M_{i}=2 m_{p}$. A high collisional case is prescribed by the same parameters except $n=6.5$ $\times 10^{19} \mathrm{~m}^{-3}$ and $T=30.8 \mathrm{eV}$ (to keep the plasma $\beta$ $=\mu_{0} n T_{e} / B^{2}$ constant). A measure for the degree of collisionality relevant for the DALF3 model is the so-called ballooning parameter $\nu_{B}$ given by 


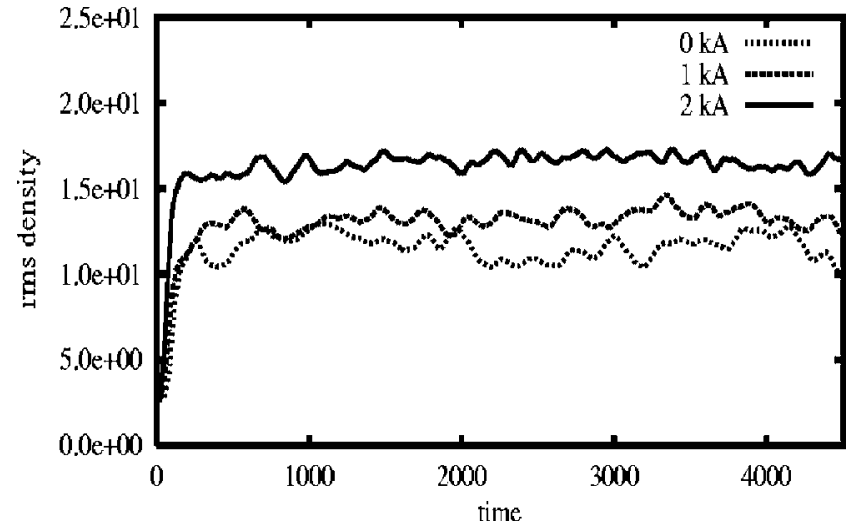

FIG. 3. Illustration of the time evolution of rms of volume-averaged density $\widetilde{n}$ (in units of $n \rho_{s} / L_{\perp}$ ) for various magnetic perturbation fields induced by an external current $I_{D}=0,1$, and $2 \mathrm{kA}$. The time is given in units of $L_{\perp} / c_{s}$.

$$
\nu_{B}=\frac{m_{e}}{m_{i}} \frac{q^{2} R_{0} \nu_{e}}{c_{s}},
$$

where $\nu_{e}$ denotes the electron-electron collision frequency. A value $\nu_{B}>1$ indicates that the dynamics is governed by ballooning activity, whereas a situation characterized by $\nu_{B}<1$ is dominated by shear-Aflvén dynamics. The values for our particular cases are $\nu_{B}=0.04$ and $\nu_{B}=1.48$ for the low and high collisional cases, respectively.

The perpendicular grid node counts are $N_{x}=64$ and $N_{y}=1024$. Consequently the pitch parameter $q$ ranges between 2.6 and 3.4, covering well the resonances of the threemode perturbations at $q=11 / 4,12 / 4$, and 13/4. The number of drift planes (grid nodes in $s$ ) is 16 . Perpendicular drift dynamics is resolved down to $2 \rho_{s}=2 \sqrt{T_{e} / M_{i}} /\left(e B_{0} / M_{i}\right)$. The time step is $\tau=0.05 L_{\perp} / c_{s}$. The initial state is a random spatial distribution of density fluctuations with a Gaussian envelope in $s$. The other dependent variables arise due to the parallel dynamics, then saturation occurs within a few $100 L_{\perp} / c_{s}$, and then the mode structure is statistically stationary after about $500 L_{\perp} / c_{s}$. This is carried to $4500 L_{\perp} / c_{s}$, with native turbulence diagnosis averaged over the interval 3000 $<c_{s} t / L_{\perp}<4500$. This procedure is applied to all the cases considered. Examples of time traces of the rms density are shown in Fig. 3.

\section{SIMULATION RESULTS}

The results of our simulations will be discussed in terms of several spatial and temporal averaged quantities. Therefore we introduce the following notations for the different kinds of averaging procedures of a scalar function $f=f(x, y, s, t)$ and their relation to the representation in Fourier components with respect to standard toroidal coordinates. A bracket like $\langle\cdots\rangle_{x},\langle\cdots\rangle_{x y}$, or $\langle\cdots\rangle_{t}$ denotes an average over the entire domain of the respective variables, i.e., here the $x$ domain, the $x y$ domain, and a certain time interval $T$. Thus it follows that

$$
\left\langle f^{2}\right\rangle_{V} \equiv \sum_{n}\left\langle f_{n}^{2}\right\rangle_{x}
$$

$$
\begin{aligned}
\left\langle f_{n}^{2}\right\rangle_{x} & \equiv \sum_{m}\left\langle\left|f_{m, n}\right|^{2}\right\rangle_{x}, \\
\left\langle f^{2}\right\rangle_{x y} & =\sum_{n} \sum_{m_{1}, m_{2}}\left\langle f_{m_{1}, n} f_{m_{2}, n}^{*}\right\rangle_{x} e^{i\left(m_{1}-m_{2}\right) \theta} .
\end{aligned}
$$

Fourier components $f_{m, n}$ are defined by

$$
f_{m, n}=\frac{1}{4 \pi^{2}} \int_{0}^{\pi} \int_{0}^{2 \pi} f e^{-i(m \theta-n \varphi)} d \theta d \varphi .
$$

For the particular radial $E \times B$ flux,

$$
\Gamma=\tilde{n} \widetilde{v}_{E, r}=-\frac{\tilde{n}}{B} \frac{\partial \widetilde{\phi}}{\partial y},
$$

where $\widetilde{v}_{E, r}=\widetilde{\mathbf{v}}_{E} \cdot \mathbf{e}_{r}$ and $\mathbf{e}_{r}$ is the unit basis vector in radial direction, one obtains

$$
\begin{aligned}
& \langle\Gamma\rangle_{V} \equiv \sum_{n}\left\langle\Gamma_{n}\right\rangle_{x}, \\
& \left\langle\Gamma_{n}\right\rangle_{x} \equiv-\frac{n K_{y}}{B} \sum_{m}\left\langle\operatorname{Im}\left\{\widetilde{n}_{m, n} \widetilde{\phi}_{m, n}^{*}\right\}\right\rangle_{x}, \\
& \langle\Gamma\rangle_{x y}=\sum_{n} \sum_{m_{1}, m_{2}} \frac{i n K_{y}}{B}\left\langle\widetilde{n}_{m_{1}, n} \widetilde{\phi}_{m_{2}, n}^{*}\right\rangle_{x} e^{i\left(m_{1}-m_{2}\right) \theta} .
\end{aligned}
$$

\section{A. Amplitudes of density and eddy strength and turbulent radial flux}

In this section we present simulation results for the density perturbations, the $E \times B$ velocity, and the resulting $E \times B$ flow. The perturbation levels and the $E \times B$ flow are increased in the presence of a magnetic perturbation field for low and high collisionalities as well, in particular, in low- $n$ modes (toroidal mode number $n<40$ ) resonant with the externally induced magnetic field. However, the response of the low collisional plasma is much stronger than that of a high collisional plasma. The radial particle flux due to the parallel mass flow along perturbed field lines $\sim n \widetilde{u}_{\|} \widetilde{\mathbf{B}}_{\perp}$ is found to be small compared to the $E \times B$ flow in all the cases considered and will not be discussed further here. Figures 4 and 5 show the $x-y$-t -averaged amplitude squares of the density $\left\langle\tilde{n}^{2}\right\rangle_{x y t}$, the $E \times B$ velocity $\left\langle\widetilde{v}_{E}^{2}\right\rangle_{x y t}$, and the $E \times B$ flux $\langle\Gamma\rangle_{x y t}$. The amplitudes of $\tilde{n}, \widetilde{v}_{E, r}$, and $\Gamma$ are given in units of $\delta n, \delta c_{s}$ and $\delta^{2} n c_{s}$, respectively, where $\delta=\rho_{s} / L_{\perp}$. As expected, the results for the unperturbed cases with $\nu_{B}=1.48$ show a more pronounced ballooning structure compared to those for $\nu_{B}=0.04$. The externally induced magnetic-field perturbations lead to an overall increase in the perturbation levels and the radial $E \times B$ flux for $\nu_{B}=0.04$ and $\nu_{B}=1.48$ as well. The response to the perturbation field does not reflect the pattern of the magnetic perturbation field with its strong localization at the high-field side at $\theta= \pm \pi$ (see Fig. 8, bottom). It is obvious that the high collisional case is less affected by the stochastic magnetic fields. While for low collisionality and a coil current of $I_{D}=2 \mathrm{kA}$ the density perturbations are increased by a factor of 7 and the $E \times B$ flux by a factor of 5 with respect to the unperturbed case, in the high collisional 

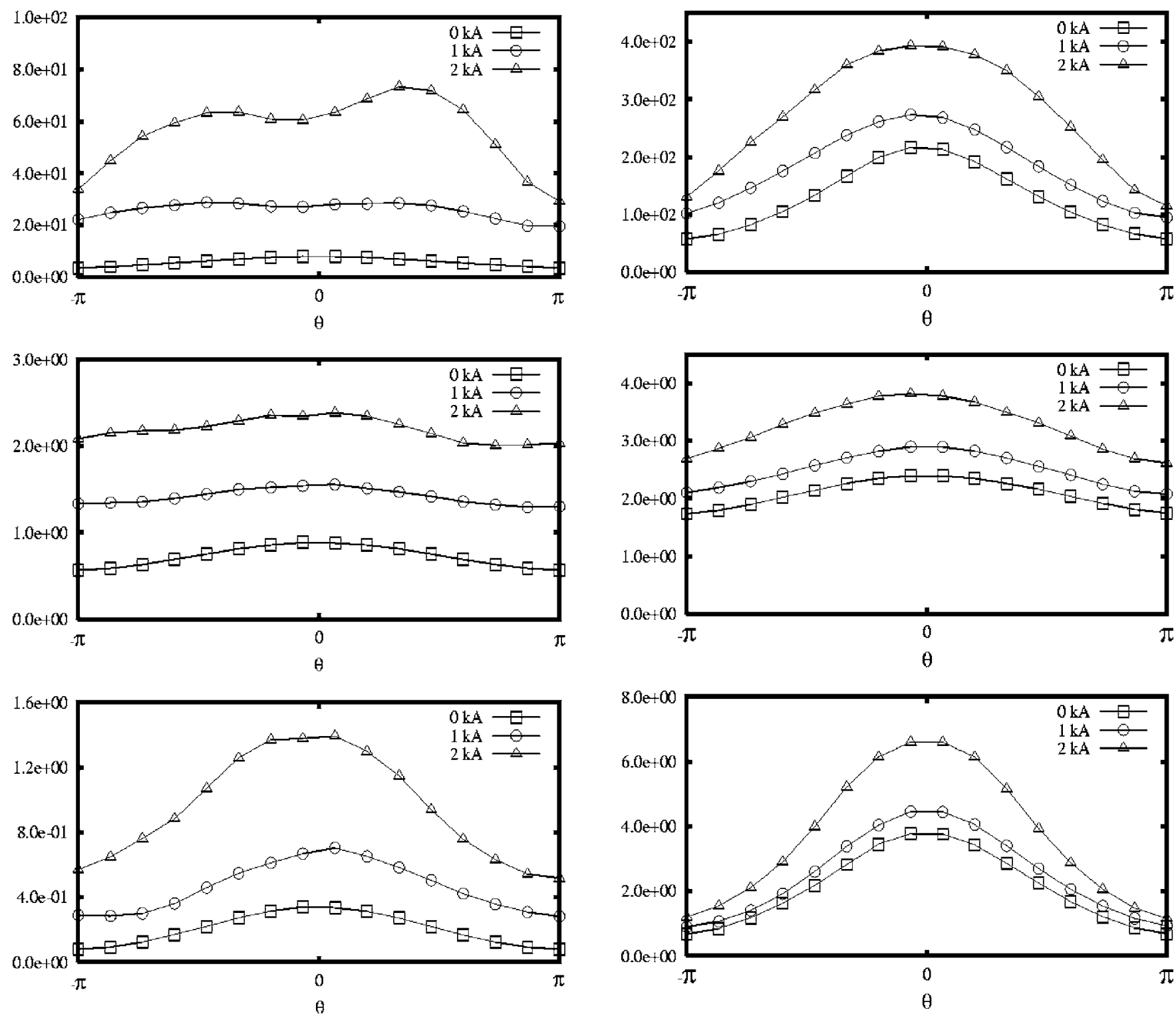

FIG. 4. Plot of density $\left\langle\widetilde{n}^{2}\right\rangle_{x y t}$ (top), $E \times B$ velocity $\left\langle\widetilde{v}_{E}^{2}\right\rangle_{x y t}$ (middle), and $E \times B$ flux $\langle\Gamma\rangle_{x y t}$ (bottom) vs poloidal angle $\theta$ for $\nu_{B}=0.04$.

case one finds an increase of about 1.5 only. In particular, for $\nu_{B}=1.48$ and $I_{D}=1 \mathrm{kA}$, i.e., for a Chirikov parameter $\sigma=1.25$, the $E \times B$ flux is quite close to that of the unperturbed case (15\% increase).

The increase of perturbation levels is also illustrated by Figs. 6 and 7 showing the averaged spectral components $\left\langle\tilde{n}_{n}^{2}\right\rangle_{x t},\left\langle\left(\widetilde{v}_{E, r}^{2}\right)_{n}\right\rangle_{x t}$ and $\left\langle\Gamma_{n}\right\rangle_{x t}$. It can be seen that the strong reaction of the perturbations is mostly pronounced in the resonant modes with toroidal mode numbers $n=4,8,12, \ldots$. The strongest effect of the magnetic perturbations is observed for mode numbers below $n=40$, where density and $E \times B$ flow are increased up to two orders of magnitude. However, one observes that also nonresonant low- $n$ modes $(n<40)$ and high- $n$ modes $(n>40)$ are affected (amplified) by the perturbation and contribute noticeably to the overall picture. Besides the similarities of the high and low collisional cases it is apparent that the low- $n$ modes have relatively larger amplitudes in the unperturbed case for

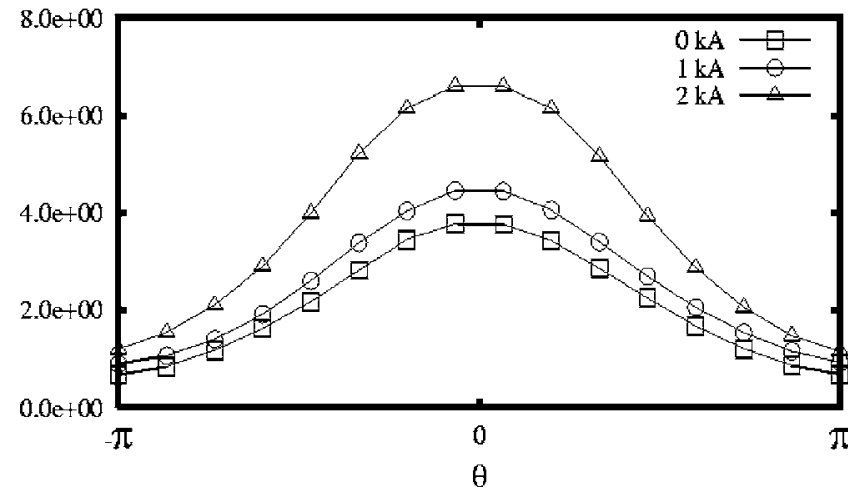

FIG. 5. Plot of density $\left\langle\widetilde{n}^{2}\right\rangle_{x y t}$ (top), $E \times B$ velocity $\left\langle\widetilde{v}_{E}^{2}\right\rangle_{x y t}$ (middle), and $E \times B$ flux $\langle\Gamma\rangle_{x y t}$ (bottom) vs poloidal angle $\theta$ for $\nu_{B}=1.48$.

$\nu_{B}=1.48$, and perturbation effects add a smaller relative contribution to the large scale eddy transport than for $\nu_{B}=0.04$.

\section{B. Magnetic flutter}

The next point we consider is the intrinsic magnetic flutter and its response to the externally induced static magnetic flutter. It is found that the intrinsic magnetic flutter is much smaller than the externally induced magnetic flutter for the perturbation fields considered, in particular, in the high collisional case.

Figure 8 shows the $x-y$ - $t$-averaged strength of the intrinsic magnetic-field perturbation $\left\langle\widetilde{\mathbf{B}}_{\text {int }} \mid\right\rangle_{x y t}$ compared to the externally induced magnetic field $\left\langle\widetilde{\mathbf{B}}_{\text {ext }} \mid\right\rangle_{x y t}$. The magnetic field in Fig. 8 is given in units of $B_{0}$, the background field. For both low and high collisionalities the intrinsic magnetic flutter is increased with increasing externally induced perturbation. Like for the density and the electrostatic potential the 

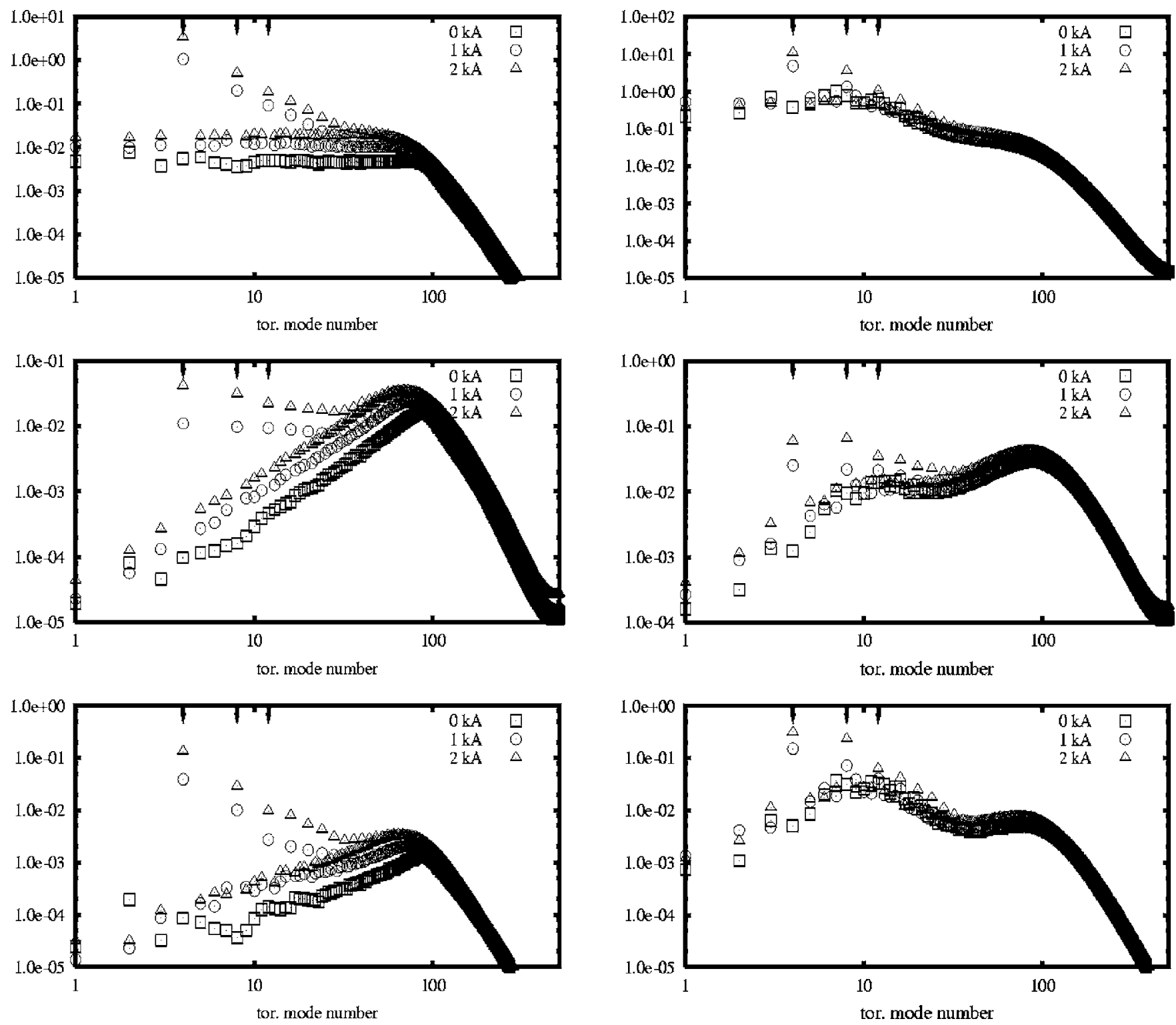

FIG. 6. Fourier amplitude plot of density $\left\langle\tilde{n}_{n}^{2}\right\rangle_{x t}$ (top), radial $E \times B$ velocity $\left\langle\left(\widetilde{v}_{E, r}\right)_{n}^{2}\right\rangle_{x t}$ (middle), and $E \times B$ flux $\left\langle\Gamma_{n}\right\rangle_{x t}$ (bottom) vs toroidal mode number $n$ for $\nu_{B}=0.04$.

effect is more pronounced for the case with low collisionality. In all the cases the maximum intrinsic response $\left\langle\widetilde{\mathbf{B}}_{\text {int }} \mid\right\rangle_{x y t}$ is at least a factor of 3 smaller than the externally induced magnetic field $\left\langle\widetilde{\mathbf{B}}_{\text {ext }} \mid\right\rangle_{x y t}$. For increasing perturbation fields the spatial structure reflects the localization at the high-field side of the imposed static perturbation. In the presence of an external perturbation the low collisional case features an intrinsic magnetic flutter a factor of 2 above that of the corresponding high collisional case.

\section{Energetics}

To get more insight into the dominant mechanisms governing the turbulent dynamics we consider in this section the different contributions to the energy theorem derived in the Appendix. The basic results found are as follows: The sound wave dynamics does not play a significant role. For low col-

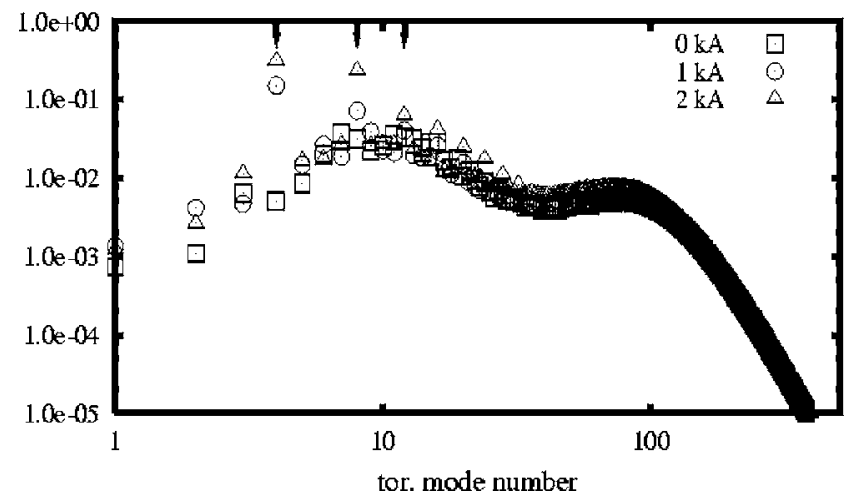

FIG. 7. Fourier amplitude plot of density $\left\langle\widetilde{n}_{n}^{2}\right\rangle_{x t}$ (top), radial $E \times B$ velocity $\left\langle\left(\widetilde{v}_{E, r}\right)_{n}^{2}\right\rangle_{x t}$ (middle), and $E \times B$ flux $\left\langle\Gamma_{n}\right\rangle_{x t}$ (bottom) vs toroidal mode number $n$ for $\nu_{B}=1.48$.

lisionality the drift-Alfvén-wave dynamics is dominating and the ballooning drive is negligible, whereas for the high collisional case the situation is reversed and ballooning turbulence becomes strongly pronounced. The low collisional case exhibits significant electromagnetic effects, whereas electromagnetic effects do not play a role for high collisionality. An externally induced magnetic perturbation field introduces additional sources for the turbulent energy which are of significant magnitude for low collisionality and act as a trigger for modes resonant with the magnetic perturbation. This effect is described by the source term $M$, representing a gain of free energy from the background density gradient due to induced magnetic flutter. Except for the additional sources $M$ the overall picture of energy flow in our model system does not change significantly for the low and high collisional cases as well. Figures 9-11 show the volume- and time-averaged contributions of the sources, sinks, and transfer terms, where 

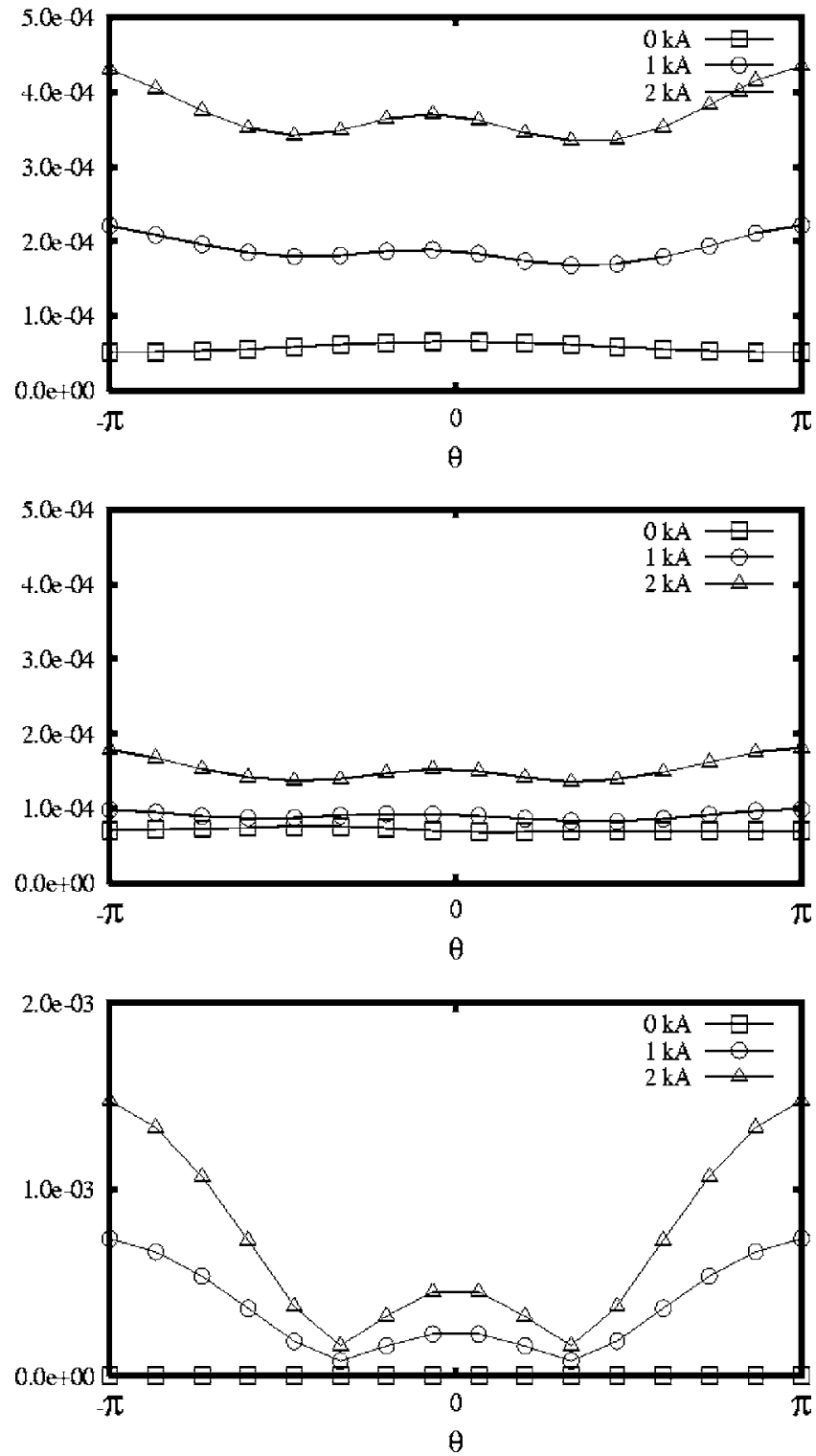

FIG. 8. Plot of intrinsic magnetic flutter $\left\langle\left|\widetilde{\mathbf{B}}_{\text {int }}\right|\right\rangle_{x y t}$ for $\nu_{B}=0.04$ (top) and $\nu_{B}=1.48$ (middle) and of the externally induced magnetic flutter $\left\langle\left|\widetilde{\mathbf{B}}_{\text {int }}\right|\right\rangle_{x y t}$ (bottom) vs poloidal angle $\theta$.

again the time averages have been computed over the last interval of $T=1500 L_{\perp} / c_{s}$ in the simulations. Figure 9 shows the contributions of the sinks and sources in terms of growth rates. For both low and high collisionalities the dominant source for the turbulent dynamics is provided by the driving term $S$, gaining energy from the background density gradient via $E \times B$ advection. The other dominant contribution is the collisional dissipation $C$. For the unperturbed cases these are the only terms of relevance and the sources $M$ and $Q$ due to the appearance of magnetic flutter do not play a role. The difference between $S$ and $C$ is positive and is compensated by numerical dissipation acting at small scales, representing viscous effects. By imposing an externally induced perturbation field this picture does not change significantly for high collisionality, but for low collisionality the contribution from $M$ becomes important, i.e., it is of the same order of magnitude as the collisional dissipation. It has to be noted that a

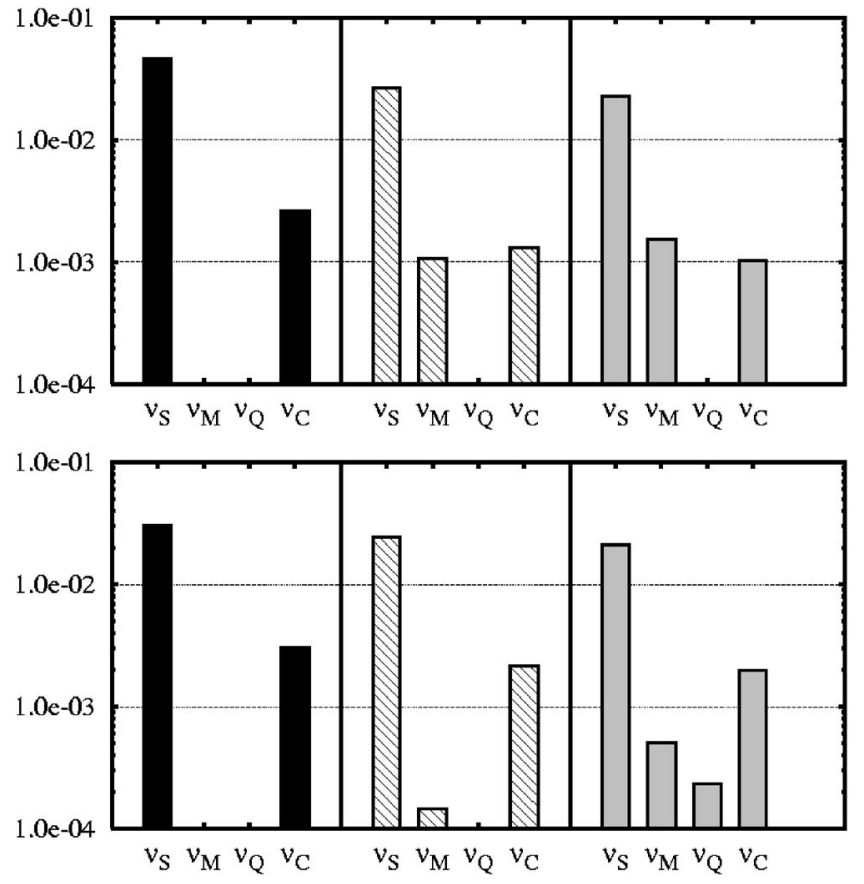

FIG. 9. Energetics plot. Shown are the $V$-t $t$-averaged growth rates $\left\langle\nu_{S}\right\rangle_{V t}$, $\left\langle\nu_{M}\right\rangle_{V t},\left\langle\nu_{Q}\right\rangle_{V t}$, and $\left\langle\nu_{C}\right\rangle_{V t}$ for $\nu_{B}=0.04$ (top) and $\nu_{B}=1.48$ (bottom) for perturbation currents $I_{D}=0 \mathrm{kA}$ (black), $I_{D}=1 \mathrm{kA}$ (striped), and $I_{D}=2 \mathrm{kA}$ (grey).

spectral analysis of the sources (not shown here) proves that the additional source $M$ acts almost solely on modes with toroidal mode number $n=4$, i.e., the resonant modes are triggered by the strong externally induced magnetic flutter. The slight decrease of $\nu_{S}$ and $\nu_{C}$ with increasing perturbation field is explained by the fact that the growth rates are defined as source strengths divided by the total free energy of the
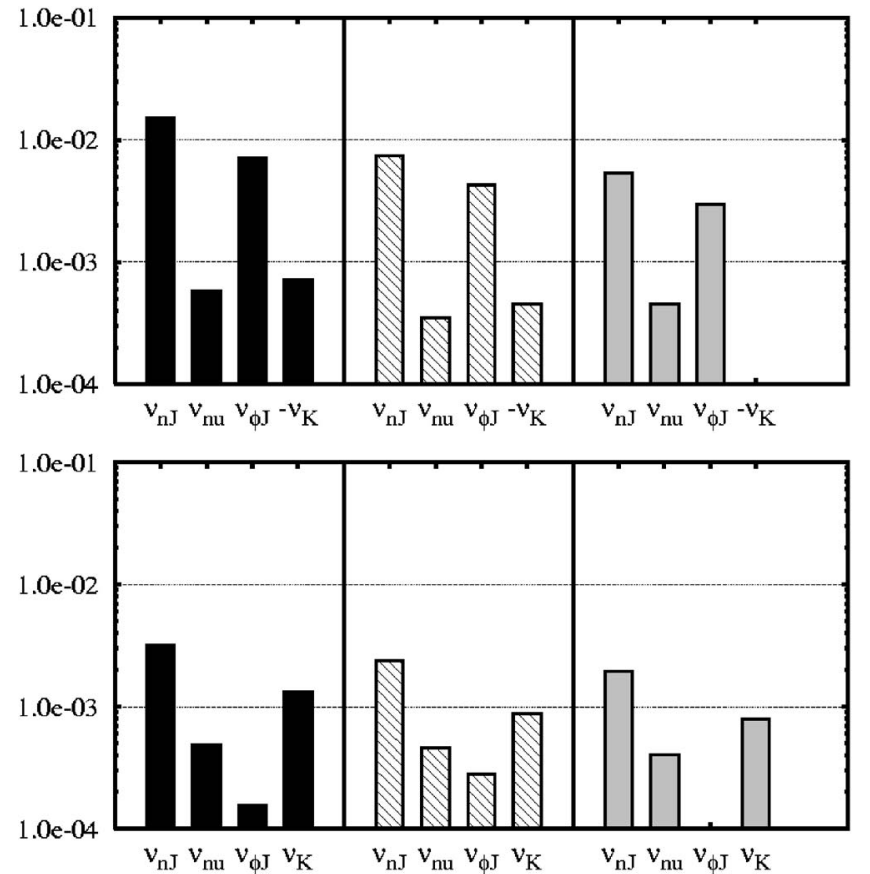

FIG. 10. Energetics plot. Shown are the $V$-t-averaged transfer rates $\left\langle\nu_{n J}\right\rangle_{V t}$, $\left\langle\nu_{n u}\right\rangle_{V t},\left\langle\nu_{\phi J}\right\rangle_{V t}$, and $\left\langle\nu_{K}\right\rangle_{V t}$ for $\nu_{B}=0.04$ (top) and $\nu_{B}=1.48$ (bottom) for perturbation currents $I_{D}=0 \mathrm{kA}$ (black), $I_{D}=1 \mathrm{kA}$ (striped), and $I_{D}=2 \mathrm{kA}$ (grey). 

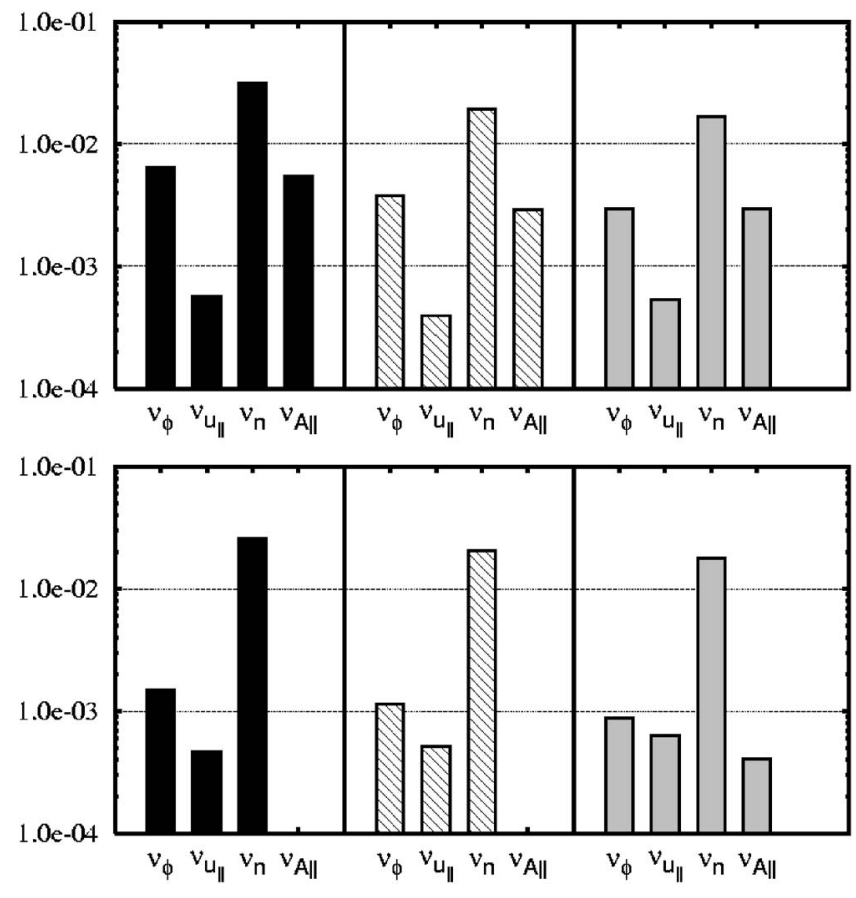

FIG. 11. Energetics plot. Shown are the $V$-t-averaged growth rates $\left\langle\nu_{\phi}\right\rangle_{V t}$, $\left\langle\nu_{u_{\|}}\right\rangle_{V t},\left\langle\nu_{n}\right\rangle_{V t}$, and $\left\langle\nu_{A_{\|}}\right\rangle_{V t}$ for $\nu_{B}=0.04$ (top) and $\nu_{B}=1.48$ (bottom) for perturbation currents $I_{D}=0 \mathrm{kA}$ (black), $I_{D}=1 \mathrm{kA}$ (striped), and $I_{D}=2 \mathrm{kA}$ (grey).

system. But this content of free energy consists of a static piece, increasing with the perturbation field, thus reducing the growth rates, if the source strengths are unchanged. The appearance of such a static contribution will be discussed more in detail in Sec. IV D.

In Fig. 10 the transfer rates are plotted. As expected the cases with $\nu_{B}=0.04$ and $\nu_{B}=1.48$ differ strongly. For low collisionality the transfer due to $T_{n J}$ and $T_{\phi J}$ dominates the internal energy transfer in the system, i.e., the transport is governed by the drift-Alfvén-wave dynamics. The ballooning effect represented by the curvature term $K$ does not contribute noticeably. For high collisionality the situation is different and $T_{n J}$ and $K$ regulate the internal energy flow, where $K$ is opposite in sign to $T_{n J}$. The increasing relevance of $K$ is reflected by the increase of $\nu_{B}$ which is actually an approximation for the ratio $K / T_{n J}$ extracted from the dissipative coupling model. ${ }^{30}$ Its approximative character is obvious by the fact that the ratio $\left|K / T_{n J}\right|$ is less than 1 for $\nu_{B}=1.48$, in contradiction to an expected ballooning dominated regime. However, the ballooning activity is strongly pronouncedalthough not dominant compared to drift-Alfvén dynamics - as can be seen also in Figs. 5 and 13 with a relative increase of amplitudes of a factor of about 4 at the low-field side at $\theta=0$. In both cases the sound wave dynamics, represented by the transfer rate $\nu_{n u}$, plays a less important role. By increasing the magnetic perturbation field no significant change in the transfer characteristics occurs, except that the ballooning drive becomes even less relevant for the low collisional case.

Finally Fig. 11 shows the growth rates of the different contributions of the free energy due to fluctuations in the four scalar quantities of the DALF3 model. All simulations done prove that the change of the total free energy is guided by the increase of density fluctuations due to the background gradient drive $S$, i.e., by the growth rate $\nu_{n}$. Again strong differences can be found between low and high collisionality scenarios. The low collisional case exhibits, in addition to $\nu_{n}$, a strong contribution from $\nu_{\phi}$, the time change in kinetic $E \times B$ energy, and-more importantly-a strong impact of electromagnetic effects represented by the rate $\nu_{A_{\|}}$. This is not seen for the high collisional case, where electromagnetic effects for $I_{D}=0 \mathrm{kA}$ and $I_{D}=1 \mathrm{kA}$ do not play a role at all and appear as small even for the highest perturbation considered, where $I_{D}=2 \mathrm{kA}$. Like for the energetic quantities above, one can observe for both collisionalities that the change of the characteristic pattern is only small with increasing perturbation field.

\section{Static versus time-dependent contributions}

In this section we address the issue of discriminating the static and time-dependent (turbulent) contributions of the results discussed in Sec. IV A. It is found that the strong increase in the amplitude levels of density and $E \times B$ velocity perturbations at resonant mode numbers presented in Sec. IV $A$ is of static nature, resulting in large scale eddies which contribute significantly to the total $E \times B$ transport. However, the turbulent $E \times B$ flux is always larger than the static piece. For low collisionality the density and velocity fluctuations are increased over a broad range of the toroidal wavenumber spectrum when the magnetic perturbation field is increased. The strong increase of turbulent $E \times B$ flux for low collisionality is mainly caused by enhanced fluctuations of high- $n$ modes, i.e., small scale eddies. For high collisionality the density fluctuations stay roughly the same for the magnetic perturbation strengths considered and despite an increase of $E \times B$ velocity fluctuations in high- $n$ modes, the turbulent $E \times B$ flux is almost unchanged because this range is separated strongly from the maximum of density fluctuations at low- $n$ modes. Before presenting the simulation results a few remarks are necessary. Actually the model equations (1)-(4) allow stationary solutions, which can be assigned to a modified background plasma, as mentioned in Sec. II A. To resolve the turbulent contributions is of particular importance for a comparison with experimental observations, which are often capable to study only time-varying perturbations in the plasma parameters, e.g., by means of reflectometry. To analyze the fluctuations, we divide the numerical solutions into a static and a time-dependent (fluctuating) piece, e.g.,

$$
\widetilde{n}(x, y, s, t)=\bar{n}(x, y, s)+\hat{n}(x, y, s, t),
$$

and discriminate $\bar{n}$ against $\hat{n}$ by averaging over a time interval of $T=1500 L_{\perp} / c_{s}$ at the end of the computations. We note that this kind of time averaging does not give the true stationary piece, fulfilling the stationary form of the model equations; rather it gives a rough picture of the ratio between static and turbulent contributions, strongly dependent on the time interval considered for averaging.

However, if we compare Figs. 4 and 5, showing the total (static and fluctuating) perturbations and radial transport, with Figs. 12 and 13, showing only the time-dependent con- 

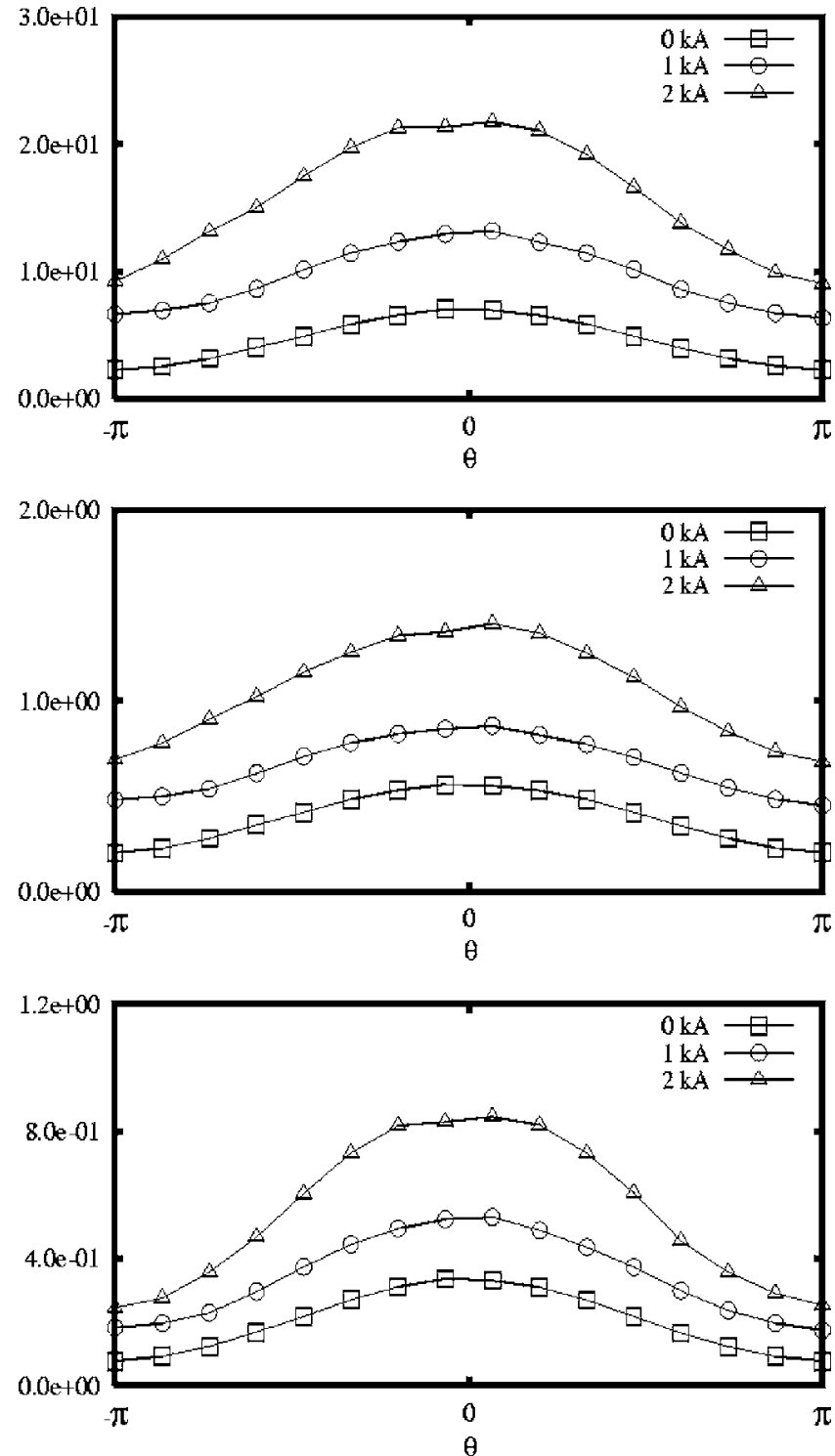

FIG. 12. Plot of density $\left\langle\widetilde{n}^{2}\right\rangle_{x y t}$ (top), $E \times B$ velocity $\left\langle\widetilde{v}_{E}^{2}\right\rangle_{x y t}$ (middle), and $E \times B$ flux $\langle\Gamma\rangle_{x y t}$ (bottom) vs poloidal angle $\theta$ for $\nu_{B}=0.04$. The "static" part (taken from time averaging) has been subtracted.

tributions as obtained by subtracting the static contributions, it is found that the strong increase in the amplitude levels of density and $E \times B$ velocity perturbations is to a large extent of static nature. The fluctuations show even more clearly the different behavior of the low and high collisional cases mentioned in the sections above: while the cases with $\nu_{B}=1.48$ indicate a maximum increase of only $10 \%-20 \%$ in the density and $E \times B$ flux, the fluctuations and the corresponding radial flux for $\nu_{B}=0.04$ are increased by a factor up to 3 and 4. In all the cases considered the turbulent piece of the $E \times B$ flux is larger than the static piece. For the unperturbed scenarios the static $E \times B$ flux is negligible compared to the turbulent transport, but for increasing perturbation fields the static part can reach half of the turbulent part. The comparison of Figs. 6 and 7 with Figs. 14 and 15 proves that the resonance effects at toroidal mode numbers $n=4,8,12, \ldots$, occurring for finite magnetic perturbation fields, are for the most part of static nature. After subtraction of the static part
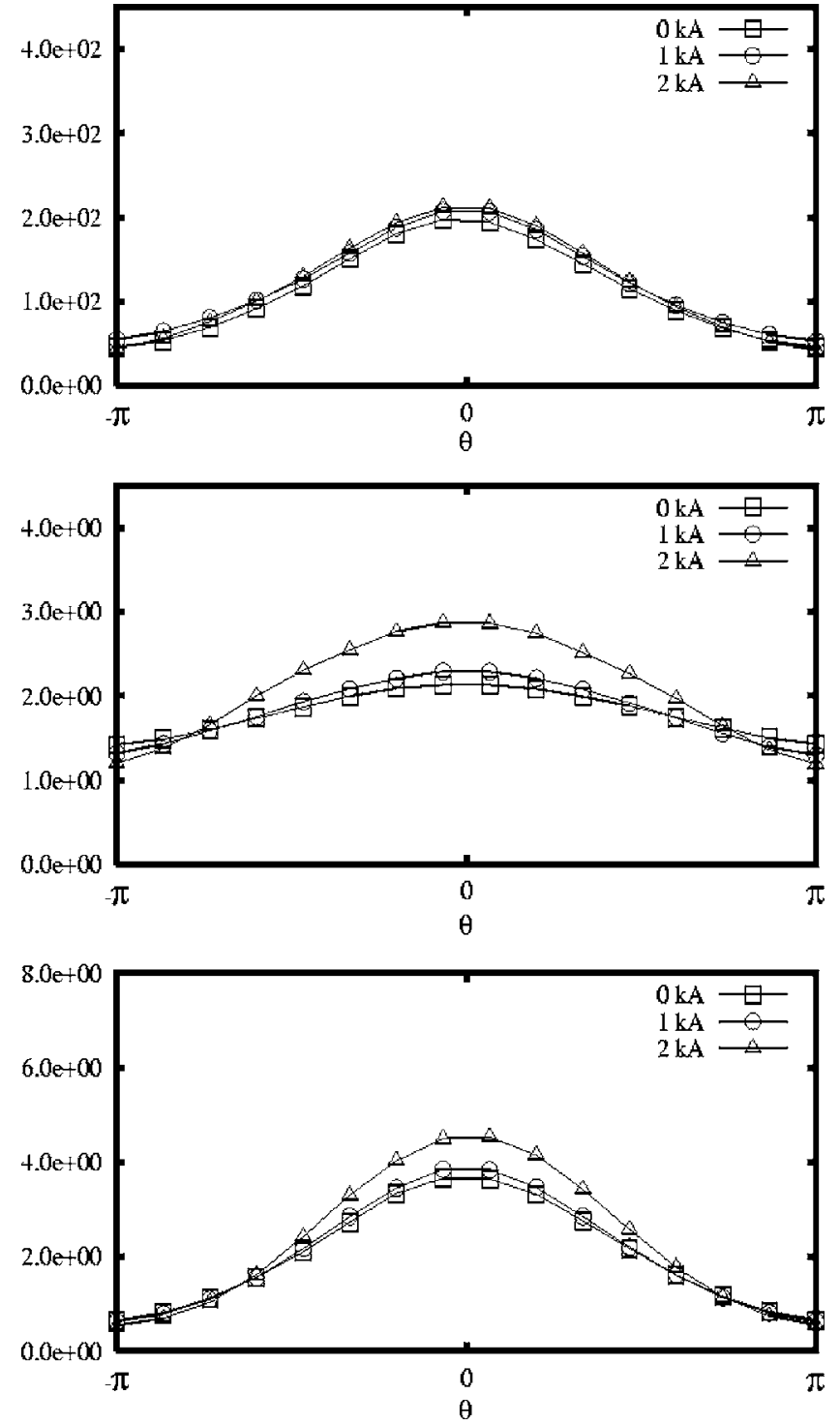

FIG. 13. Plot of density $\left\langle\widetilde{n}^{2}\right\rangle_{x y t}$ (top), $E \times B$ velocity $\left\langle\widetilde{v}_{E}^{2}\right\rangle_{x y t}$ (middle), and $E \times B$ flux $\langle\Gamma\rangle_{x y t}$ (bottom) vs poloidal angle $\theta$ for $\nu_{B}=1.48$. The "static" part (taken from time averaging) has been subtracted.

reminiscents of the resonances are visible for $\nu_{B}=0.04$, and for $\nu_{B}=1.48$ those resonances are almost vanished. The reminiscents of resonances might occur due to the approximative calculation of the static part. It is advantageous to continue the spectral analysis of the fluctuations and the turbulent $E$ $\times B$ flux by the use of the half-logarithmic plots in Figs. 16 and 17 (showing the same results as Figs. 14 and 15). These demonstrate that the increase in the turbulent radial flux for the low collisional case with $\nu_{B}=0.04$ is caused mainly by enhanced fluctuations of density and $E \times B$ velocity in high$n$ modes, i.e., small scale eddies. For the high collisional case the situation is different. Although there is also an increase in $\tilde{v}_{E, r}$ fluctuations for high- $n$ modes, the density fluctuations are strongest for low- $n$ modes. Due to numerical errors caused by the averaging procedure to obtain the static part, the low- $n$ part of the spectrum is quite noisy and there is no clear tendency observable, whether low- $n$ modes are damped or amplified. It looks rather like a zero net effect, even 

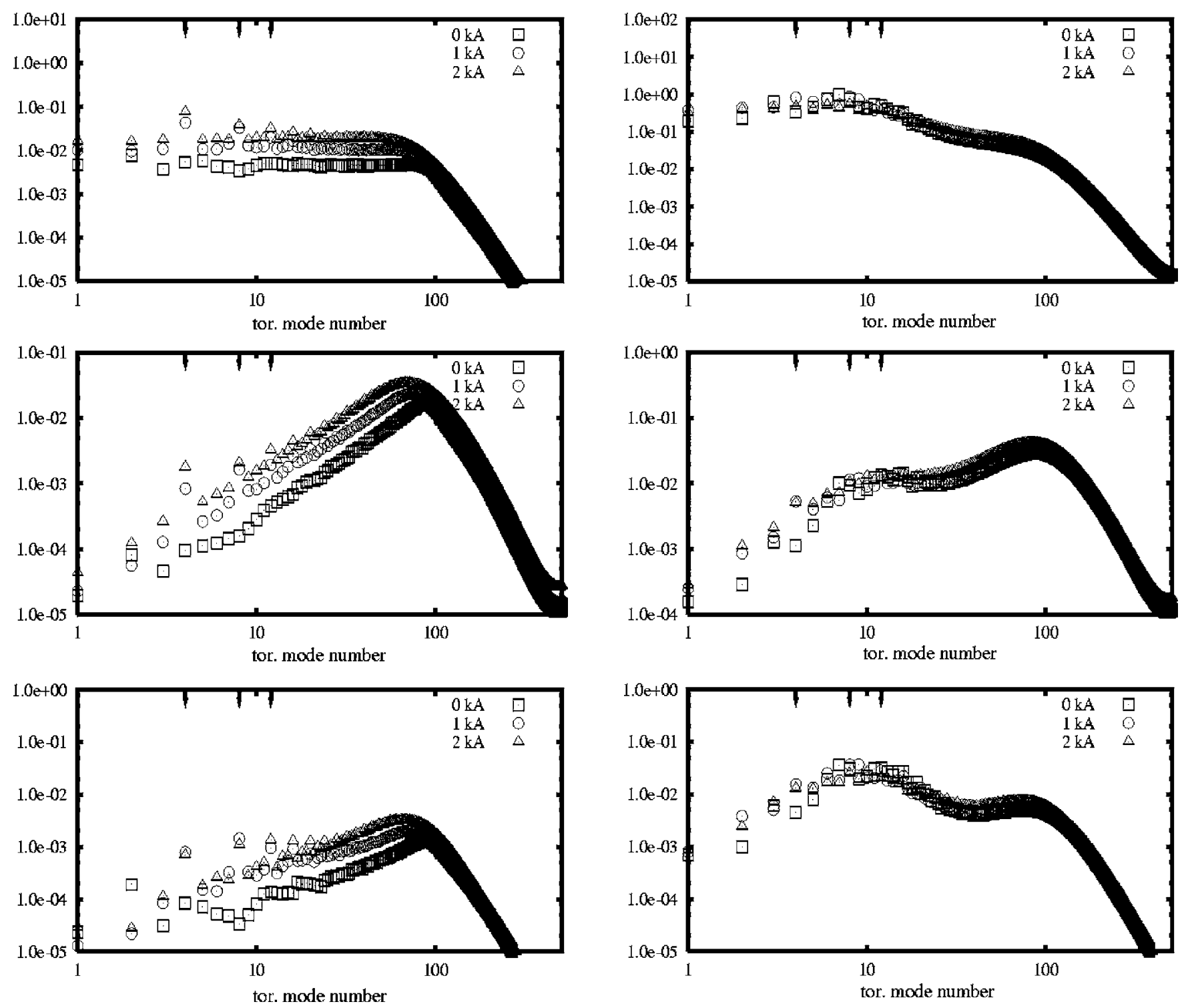

FIG. 14. Fourier amplitude plot of density $\left\langle\tilde{n}_{n}^{2}\right\rangle_{x t}$ (top), radial $E \times B$ velocity $\left\langle\left(\widetilde{v}_{E, r}\right)_{n}^{2}\right\rangle_{x t}$ (middle), and $E \times B$ flux $\left\langle\Gamma_{n}\right\rangle_{x t}$ (bottom) vs toroidal mode number $n$ for $\nu_{B}=0.04$. The "static" part (taken from time averaging) has been subtracted.

though almost all amplitudes for $n<40$ seem to be damped for $\nu_{B}=1.48$ and $I_{D}=2 \mathrm{kA}$. As a consequence the radial $E \times B$ flux for high collisionality is carried to a large extent by low- $n$ modes and does not exhibit a significant change in the presence of an ergodic magnetic perturbation.

\section{SUMMARY AND DISCUSSION}

Three-dimensional nonlinear electromagnetic fluid drift transport has been simulated using the DALF3 code. We considered a thin toroidal annulus centered around the $q=3$ surface and computed the small scale dynamics of the scalar quantities density $\widetilde{n}$, electrostatic potential $\widetilde{\phi}$, parallel ion velocity $\widetilde{u}_{\|}$, and magnetic potential $\widetilde{A}_{\|}$for plasma parameters representative for TEXTOR-DED discharges. To study the effect of ergodized magnetic fields on the small scale dynamics, externally induced three-mode perturbation fields, con-

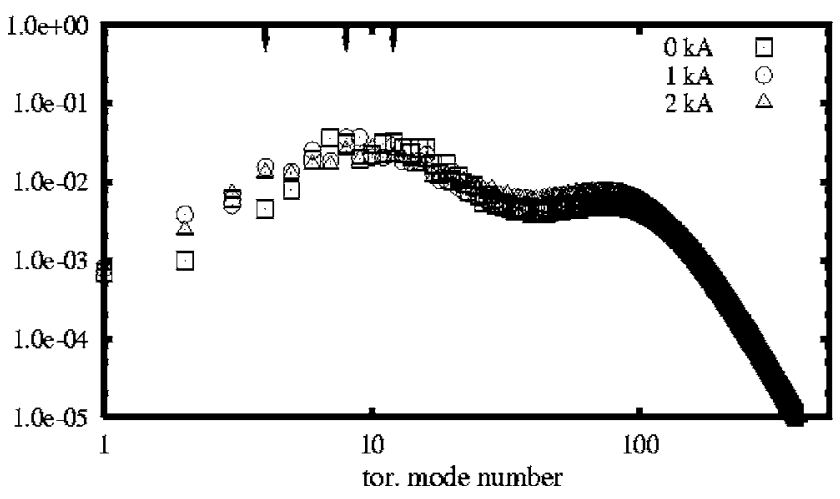

FIG. 15. Fourier amplitude plot of density $\left\langle\tilde{n}_{n}^{2}\right\rangle_{x t}$ (top), radial $E \times B$ velocity $\left\langle\left(\widetilde{v}_{E, r}\right)_{n}^{2}\right\rangle_{x t}$ (middle), and $E \times B$ flux $\left\langle\Gamma_{n}\right\rangle_{x t}$ (bottom) vs toroidal mode number $n$ for $\nu_{B}=1.48$. The "static" part (taken from time averaging) has been subtracted.

sisting of 11/4, 12/4, and 13/4 modes, have been imposed introducing a stochastic magnetic structure in the plasma. Simulation results for different combinations of different external coil currents, representing Chirikov parameters of $\sigma=1.25$ and $\sigma=1.78$, and different collisionalities of the background plasma, corresponding to ballooning parameters $\nu_{B}=0.04$ and $\nu_{B}=1.48$, have been compared with unperturbed turbulence simulations. Averaged poloidal profiles of density, $E \times B$ velocity, and $E \times B$ flux have been presented, accompanied by a spectral analysis with respect to toroidal mode numbers. By means of time averaging of the saturated turbulent state, fluctuating and static pieces of the transport have been separated approximately. The energy flow of the model system has been analyzed by means of an appropriate energy theorem to study the turbulence characteristics. The results can be summarized as follows:

(1) The static ergodization fields introduce a strong static 

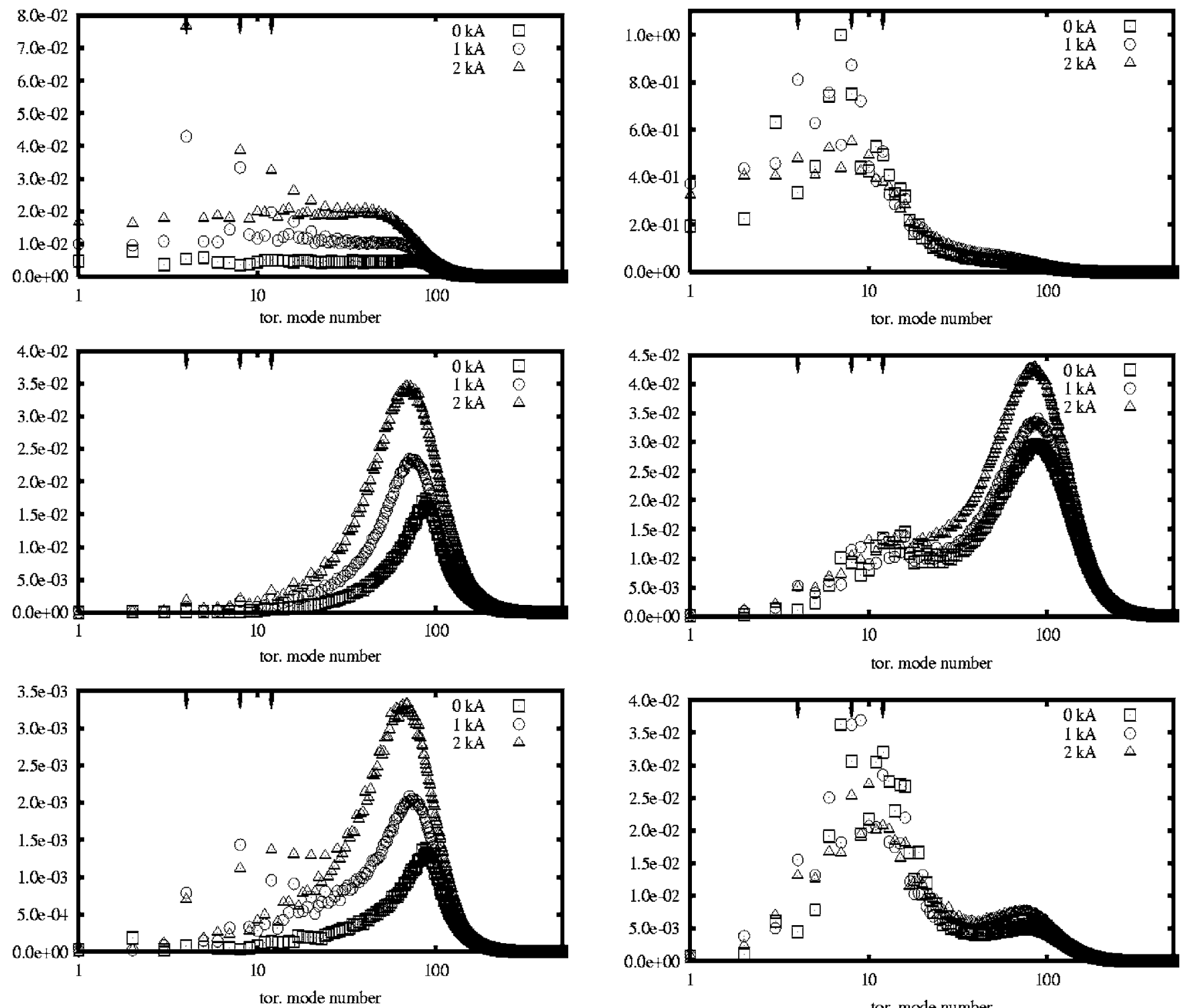

FIG. 16. Fourier amplitude plot of density $\left\langle\tilde{n}_{n}^{2}\right\rangle_{x t}$ (top), radial $E \times B$ velocity $\left\langle\left(\widetilde{v}_{E, r}\right)_{n}^{2}\right\rangle_{x t}$ (middle), and $E \times B$ flux $\left\langle\Gamma_{n}\right\rangle_{x t}$ (bottom) vs toroidal mode number $n$ for $\nu_{B}=0.04$. The "static" part (taken from time averaging) has been subtracted.

perturbation in all scalar fields of the four-field model considered. This happens predominantly in modes resonant with the externally induced perturbation and its harmonics, i.e., with toroidal mode numbers $n=4,8,12, \ldots$, and is more pronounced for a low collisional plasma than for a high collisional plasma.

(2) Even though the parallel ion velocity $\widetilde{u}_{\|}$builds up significant static profiles too, this does not contribute noticeably to the radial particle transport via the corresponding transport $\sim n \widetilde{u}_{\|} \widetilde{\mathbf{B}}_{\perp}$. This parallel mass flow along the perturbed field lines is much smaller than the $E \times B$ transport in our calculations.

(3) The intrinsic magnetic flutter responding to the externally induced magnetic flutter is also for the most part static and follows the spatial structure of the perturbation field. The intrinsic magnetic flutter is always smaller than the externally induced one, but can reach

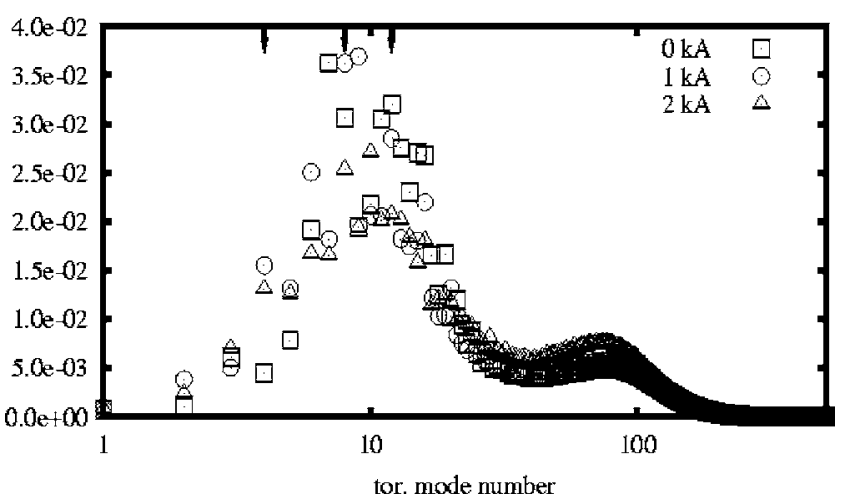

FIG. 17. Fourier amplitude plot of density $\left\langle\widetilde{n}_{n}^{2}\right\rangle_{x t}$ (top), radial $E \times B$ velocity $\left\langle\left(\widetilde{v}_{E, r}\right)_{n}^{2}\right\rangle_{x t}$ (middle), and $E \times B$ flux $\left\langle\Gamma_{n}\right\rangle_{x t}$ (bottom) vs toroidal mode number $n$ for $\nu_{B}=1.48$. The "static" part (taken from time averaging) has been subtracted.

values up to about $30 \%$ of the perturbation fields for low collisionality. For high collisionality the intrinsic flutter is by a factor of 2 smaller than for low collisionality.

(4) The analysis of energetics shows that characteristic features of the energy flow are preserved when increasing the perturbation field strength. The sound wave dynamics does not play a significant role. The energyconserving transfer terms due to sound waves, kinetic shear Alfvén waves, and curvature effects show the typical pattern of a strong ballooning drive for $\nu_{B}=1.48$ and drift-Alfvén-wave-dominated turbulence for $\nu_{B}=0.04$. The rate of change of the energies corresponding to the four turbulent scalar quantities proves that for low collisionality the electromagnetic effects play an important role, whereas they are negligible for high collisionality. An additional source appears for the low collisional case 
TABLE I. Volume- and time-averaged radial $E \times B$ fluxes, separated into static and turbulent contributions. The flux $\Gamma$ is given in units of $\delta^{2} n c_{s}$ and the coil current $I_{D}$ in units of kA.

\begin{tabular}{cccc}
\hline \hline$\nu_{B}$ & $I_{D}$ & $\langle\Gamma\rangle_{V t}$ stat & $\langle\Gamma\rangle_{V t}$ turb \\
\hline 0.04 & 0 & 0.00 & 0.20 \\
0.04 & 1 & 0.12 & 0.34 \\
0.04 & 2 & 0.40 & 0.54 \\
1.48 & 0 & 0.07 & 2.00 \\
1.48 & 1 & 0.45 & 2.10 \\
1.48 & 2 & 1.38 & 2.30 \\
\hline \hline
\end{tabular}

via the driving term $M \sim\left\langle\widetilde{J}_{\|} \nabla_{\|} n\right\rangle_{V}$, which triggers strongly the modes with $n=n_{0}$, where $n_{0}=4$ is the central toroidal mode number of the perturbation considered.

(5) The time-dependent density perturbations (fluctuations) behave completely different for the low and high collisional cases in the presence of an ergodic magnetic field. The density fluctuations for $\nu_{B}=1.48$ are only slightly increased (and this increase is likely to be smaller than the error bar due to the approximative separation of the static and fluctuating pieces), but the density fluctuations for $\nu_{B}=0.04$ are increased by a factor of 5 for $\sigma=1.78$. A similar result has been found for the turbulent radial $E \times B$ flux: only a slight increase for high collisionality and an increase by a factor of 3 for low collisionality. For both collisionalities considered a significant increase of $\widetilde{v}_{E}$ fluctuations appears for modes with $n>40$. For $\nu_{B}=0.04$ also the density fluctuations for these mode numbers are of considerable magnitude, but for $\nu_{B}=1.48$ the density fluctuations have their largest amplitudes in the range of $n<10$. Therefore the turbulent $E \times B$ flux is increased in the low collisional plasma and is carried predominantly by small scale eddies. In the high collisional case, however, only large scale eddies $(n<40)$ contribute significantly to the turbulent transport, and these are almost unchanged by a magnetic perturbation field.

(6) The turbulent $E \times B$ flux is always larger than the static piece. An overview of the different contributions is given in Table I, listing the volume- and time-averaged $E \times B$-fluxes.

The observations can be cast in the following picture: Static ergodic magnetic fields cause strong static profiles in the plasma parameters, reflecting the spatial symmetry of the perturbation field. These static structures are increased in amplitude with increasing magnetic perturbation and exhibit a significant radial $E \times B$ transport due to large scale eddies. On the other hand also the fluctuation energy and the related turbulent transport are affected by the stochastization field, but very differently for low and high collisionalities. For low collisionality an additional source for the turbulent energy appears and increases the fluctuation amplitudes and the turbulent $E \times B$ flux as well. For high collisionality this does not appear. There the additional source does not play a role and correspondingly the fluctuation amplitudes are rather unchanged.
This raises the following questions:

(1) Why are the static perturbations increased by the perturbation field?

(2) Why does the source $M$ contribute significantly only for the low collisional (electromagnetic) case and not for the high collisional (electrostatic) case?

(3) Why does the reduced MHD model of Refs. 18 and 19 give different results? Like in this work it has been found that significant static structures appear, but the density fluctuations are reduced and the total radial $E \times B$ transport (static+turbulent) is unchanged.

To discuss the first question, the buildup of static profiles, we try to find a simple relation between the magnetic perturbation and the static solutions of the model equations. Therefore we insert explicitly the static and time-dependent pieces [see Eq. (34)] of the scalar fields into the model equations (1)-(4) and consider their statistical time average. To simplify matters we neglect all radial derivatives of the scalar fields, except for the magnetic potential $\bar{A}_{\|}$, which isaccording to the simulation results-assumed to be the static externally induced perturbation field only, i.e., an intrinsic magnetic flutter is neglected. Only terms of the first order in the perturbations $\bar{n}, \bar{\phi}, \bar{J}_{\|}$, and $\bar{u}_{\|}$are kept, thus retaining the nonlinear terms including the magnetic potential, which is approximated by a single mode perturbation resonant at $q_{0}=m_{0} / n_{0}$ and with exponential radial dependence, i.e.,

$$
\bar{A}_{\|}=-A_{0} \cos \left(m_{0} \theta-n_{0} \varphi\right), \quad \frac{\bar{A}_{\|}}{\partial r}=\frac{\bar{A}_{\|}}{L} .
$$

In toroidal coordinates and using the scaling $\bar{\phi} \rightarrow T_{e} \bar{\phi} / e$, $\bar{n} \rightarrow n \bar{n}, \quad \bar{J}_{\|} \rightarrow \delta e n c_{s} \bar{J}_{\|}$, and $\bar{u}_{\|} \rightarrow \delta c_{s} \bar{u}_{\|}$with $\delta=\rho_{s} / L_{\perp}$, one obtains

$$
\begin{aligned}
& \nabla_{\|} \bar{u}_{\|}=-\frac{1}{r_{0}} \frac{\partial \bar{\phi}}{\partial \theta}+\frac{2 L_{\perp}}{R_{0}} \frac{\cos \theta}{r_{0}} \frac{\partial \bar{\phi}}{\partial \theta}, \\
& \nabla_{\|} \bar{\phi}=-\delta \frac{\nu_{B}}{1.96 q^{2} R_{0}} \bar{J}_{\|}, \\
& \nabla_{\|} \bar{n}=\frac{1}{B_{0} L_{\perp}} \frac{1}{r_{0}} \frac{\partial \bar{A}_{\|}}{\partial \theta}, \\
& \nabla_{\|} \bar{J}_{\|}=\frac{2 L_{\perp}}{R_{0}} \frac{\cos \theta}{r_{0}} \frac{\partial \bar{n}}{\partial \theta},
\end{aligned}
$$

which are the simplified versions of the difference between Eqs. (1) and (4), the sum of Eqs. (2) and (3), Eq. (3), and Eq. (4), respectively. According to the assumptions made the parallel gradient is given by

$$
\nabla_{\|}=\frac{1}{q R_{0}}\left(\frac{\partial}{\partial \theta}+q \frac{\partial}{\partial \varphi}\right)-\frac{\bar{A}_{\|}}{B_{0} L} \frac{1}{r_{0}} \frac{\partial}{\partial \theta} .
$$

It is apparent that due to the finite static magnetic perturbation field in Eq. (38), a zero static solution is not possible. Inspecting Eq. (38) by means of a Fourier transform $\bar{n}=\Sigma_{m, n} \bar{n}_{m, n} \exp [i(m \theta-n \varphi)]$ one finds 


$$
\begin{aligned}
& \frac{m-n q}{q R_{0}} \bar{n}_{m, n}+\frac{A_{0}}{2 B_{0} L} \frac{m-m_{0}}{r_{0}} \bar{n}_{m-m_{0}, n-n_{0}} \\
& \quad+\frac{A_{0}}{2 B_{0} L} \frac{m+m_{0}}{r_{0}} \bar{n}_{m+m_{0}, n+n_{0}} \\
& =-\frac{A_{0}}{2 B_{0} L_{\perp}} \frac{m}{r_{0}}\left(\delta_{m, m_{0}}+\delta_{m,-m_{0}}\right)
\end{aligned}
$$

and this can be written as a recurrence relation for the resonant modes $\bar{n}_{k}=\bar{n}_{k m_{0}, k n_{0}}$,

$$
\begin{aligned}
& k_{\|} \bar{n}_{1}+2 b \bar{n}_{2}=-c, \\
& (k-1) b \bar{n}_{k-1}+k k_{\|} \bar{n}_{k}+(k+1) b \bar{n}_{k+1}=0,
\end{aligned}
$$

where $k=2,3, \ldots$ and

$$
k_{\|}=\frac{n_{0}\left(q_{0}-q\right)}{q R_{0}}, \quad b=\frac{m_{0}}{r_{0}} \frac{A_{0}}{2 B_{0} L}, \quad c=b \frac{L}{L_{\perp}} .
$$

The recurrence relation [Eqs. (42)] can be interpreted as a trigger for the buildup of static profiles in the resonant mode and its harmonics. The trigger of the static modes is provided by the radial perturbation field (represented by the coefficient c) and the mode coupling by the poloidal perturbation field (represented by $b$ ). In particular, for $k_{\|}=0$ and $L / L_{\perp} \approx 1$, one obtains $\quad \bar{n}_{2 k+1}=(-1)^{k} /(k+1) \bar{n}_{1}$ and $\bar{n}_{2 k}=-\frac{3}{2}(-1)^{k} /(2 k+1)$, where $k=1,2, \ldots$. If now $\bar{n}_{1} \gtrsim \bar{n}_{2}$ this resembles very much the results for the resonant static modes in the density shown in Figs. 6 and 7, with amplitudes in the order of magnitude of the background density and a strong decay with increasing toroidal mode number. This gives a rough explanation of the appearance of the strong static modes in resonant mode numbers. It has to be noted that a similar explanation can be found even if the sound wave dynamics is neglected.

The second question, why the source $M$ contributes for low but not for high collisionality, can be discussed by inspecting Ohm's law [Eq. (2)] and the corresponding part of the energy theorem [Eq. (A10) in the Appendix]. Equation (2) describes essentially the evolution of the magnetic perturbation field and thus a perturbation in the current density $\widetilde{J}_{\|}$. The term $M$ in the energy balance tends to increase the energy stored in the magnetic flutter and consequently the current density for the main resonant mode. If the collisionality is high this source is compensated completely and this mode cannot grow strongly. Also the other contributions in Eq. (A10) are compensated by the resistive term, thus leading to an electrostatic behavior. For low collisionality this mechanism does not work anymore, i.e., before the current is strong enough such that $C$ can cancel the other effects, the energy transfer due to drift Alfvén waves (via $T_{\phi J}$ ) offers a channel to pass the energy gained via the term $M$ to the system. This allows $\widetilde{J}_{\|}$and $M$ to have a larger value compared to the high collisional case. For increasing magnetic perturbation this should be more pronounced, even for high collisionality, and, in fact, we have observed (see Figs. 9 and 11) an increase of $M$ for $\nu_{B}=1.48$ and $I_{D}=2 \mathrm{kA}$, accompanied by some electromagnetic signature, i.e., finite value of $\nu_{A_{\|}}$.
The third question, concerning the discrepancy of the results presented here with the results of Refs. 18 and 19, can be discussed also by an energy balance for the different model systems. First we recall in our notation the electrostatic drift-resistive ballooning limit of our model neglecting here the $\nabla_{\|} B$ contributions in the parallel derivatives to simplify the formulas and the discussion,

$$
\begin{aligned}
& \frac{d \tilde{n}}{d t}=-\widetilde{\mathbf{v}}_{E} \cdot \nabla n+\frac{1}{e} \nabla_{\|} \widetilde{J}_{\|}+n \mathcal{K}(\widetilde{\phi})-\frac{T_{e}}{e} \mathcal{K}(\widetilde{n}), \\
& \eta_{\|} \widetilde{J}_{\|}=\frac{T_{e}}{n e} \nabla_{\|}(n+\widetilde{n})-\nabla_{\|} \widetilde{\phi}, \\
& \frac{n M_{i}}{B^{2}} \frac{d}{d t} \nabla_{\perp}^{2} \widetilde{\phi}=\nabla_{\|} \widetilde{J}_{\|}-T_{e} \mathcal{K}(\widetilde{n}) .
\end{aligned}
$$

The energy diagnostics in our simulations proved that this is a reasonable reduction for the high collisional case (sound wave dynamics and electromagnetic effects are negligible). Thus the third term in Eq. (44) can be expressed by

$$
\frac{1}{e} \nabla_{\|} \widetilde{J}_{\|}=\frac{T_{e}}{n e^{2} \eta_{\|}} \nabla_{\|}^{2}(n+\tilde{n})-\frac{1}{e \eta_{\|}} \nabla_{\|}^{2} \tilde{\phi} .
$$

The energy theorem for the model equations (44)-(46) reads as $\nu_{n}=\nu_{S}-\nu_{n J}-\nu_{K}, \nu_{\phi}=\nu_{\phi J}+\nu_{K}$, and $\nu_{M}+\nu_{n J}-\nu_{\phi J}-\nu_{C}=0$, giving a temporal change in the total free energy of

$$
\frac{\partial \ln \widetilde{E}}{\partial t}=\nu_{S}-\nu_{C}+\nu_{M}
$$

Thus like before [compare with Eq. (A25)] the background density source, the resistive dissipation, and the source due to magnetic flutter govern the energy content of the system, just the source term $\nu_{Q}$ is removed by the neglect of sound waves. Now the RBM3D model of Refs. 18 and 19 in our notation appears as

$$
\begin{aligned}
& \frac{d \tilde{n}}{d t}=-\widetilde{\mathbf{v}}_{E} \cdot \nabla n+n \mathcal{K}(\widetilde{\phi})+\chi_{\|} \nabla_{\|}^{2}(n+\widetilde{n}), \\
& \eta_{\|} \widetilde{J}_{\|}=-\nabla_{\|} \widetilde{\phi}, \\
& \frac{n M_{i}}{B^{2}} \frac{d}{d t} \nabla_{\perp}^{2} \widetilde{\phi}=\nabla_{\|} \widetilde{J}_{\|}-T_{e} \mathcal{K}(\tilde{n}) .
\end{aligned}
$$

Here we did not write explicitly the additional viscous terms which provide dissipation on the perpendicular subscales. Such terms are always present and necessary in turbulence simulations, and in the DALF3 algorithm these are provided by numerical dissipation. It is also important to note that the model equations in Refs. 18 and 19 are written for the electric potential and the pressure perturbations $\widetilde{\phi}$ and $\tilde{p}$. But due to their equal treatment of pressure and density fluctuations in the interpretation of their results it is justified to consider the density equation [Eq. (49)] for the following discussion. Except the last term in Eq. (49), i.e., $\chi_{\|} \nabla_{\|}^{2}(n+\widetilde{n})$, where $\chi_{\|}$is the classical thermal heat conductivity, Eqs. (49)-(51) are identical with the resistive MHD model, of which energy balance is given by $\nu_{n}=\nu_{S}-\nu_{K}, \nu_{\phi}=\nu_{\phi J}+\nu_{K}$, and $\nu_{\phi J}+\nu_{C}$ 
$=0$. It follows that the temporal change of the total free energy in the RBM3D model is given by

$$
\frac{\partial \ln \widetilde{E}}{\partial t}=\nu_{S}-\nu_{C}+\frac{1}{\widetilde{E}} \int \frac{T_{e} \chi_{\|}}{n} \widetilde{n} \nabla_{\|}^{2}(n+\widetilde{n}) d V .
$$

Neglecting surface terms the integral can be rewritten by the use of

$$
\int \widetilde{n} \nabla_{\|}^{2}(n+\widetilde{n}) d V=-\int\left(\nabla_{\|} \tilde{n}\right)^{2} d V-\int \nabla_{\|} \tilde{n} \nabla_{\|} n d V .
$$

The last integral in Eq. (53) can be neglected due to the same arguments used in the discussion of the source term $M$ for high collisionality. Thus we are left with

$$
\frac{\partial \ln \widetilde{E}}{\partial t}=\nu_{S}-\nu_{C}-\frac{1}{\widetilde{E}} \int \frac{T_{e} \chi_{\|}}{n}\left(\nabla_{\|} \widetilde{n}\right)^{2} d V,
$$

i.e., due to the neglect of the coupling between the electric field and pressure forces acting on the electrons the source term $M$ is vanished, but still the main source $S$ and the resistive dissipation are present. As discussed above, the source term $M$ is also not important in our simulations for high collisionality. But the last term in Eq. (54) indicates an important difference between our model [see Eq. (A25)] and the RBM3D model. Compared to the model of this work the energy balance [Eq. (54)] contains an additional negative definite term offering an additional channel for the loss of turbulent energy by damping of density fluctuations. This is of particular importance if externally induced magnetic perturbations come into play. Due to the increase of the parallel gradient $\nabla_{\|} \tilde{n}$, then also containing a radial derivative, the damping of density fluctuations is increased with increasing magnetic perturbations and this is what has been found in the previous simulations of Refs. 18 and 19. How does this difference in the models match the statement made in the Introduction that the RBM3D model is wholly contained in our model [Eqs. (1)-(4)]? By noting that

$$
\chi_{\|}=\frac{3.2}{1.96} \frac{T_{e}}{n e^{2} \eta_{\|}} \approx \frac{T_{e}}{n e^{2} \eta_{\|}},
$$

it can be seen from Eq. (47) that the dissipative term $\chi_{\|} \nabla_{\|}^{2}(n+\tilde{n})$ of the RBM3D model also appears in the density equation [Eq. (44)] of the drift-resistive ballooning limit, but there it is part of the drift-Alfvén dynamics and does not change the total energy balance, i.e., the corresponding term $T_{n J}$ cancels out. Thus the connection between the different models can be summarized as follows: the RBM3D model can be extracted from our model by the neglect of sound waves and electromagnetic effects and using the MHD assumption. The remaining parallel density dissipation is found to be a part of the drift-Alfvén dynamics in our model. Consequently the RBM3D model is, in fact, a subset of our model, but an important difference occurs by the incorporation of the full drift-Alfvén dynamics in our model taking into account also counteracting terms which compensate the parallel density dissipation. In agreement with the energy balances [Eqs. (A25) and (54)] this gives an almost unchanged level of density fluctuations for high collisionality in our simulations, whereas the previous results of Refs. 18 and 19 show that a certain decrease of density fluctuations occurs for increasing magnetic perturbations.

\section{CONCLUSION}

Static ergodic magnetic fields have a twofold impact on the edge plasma transport of present day tokamak devices: on one hand they induce pronounced static profile deformations in the density, electrostatic potential, etc., providing a significant radial $E \times B$ transport due to large scale eddies, which can be of the same order as the turbulent transport in the unperturbed plasma, or even stronger. The radial transport due to mass flow along the perturbed field lines is found to be small compared to the $E \times B$ flow in our nonlinear simulations, in agreement with simple estimates given in Refs. 15 and 16. Besides the static large scale transport, an impact on high- $n$ modes (small scale eddies) has been observed, which increases the turbulent $E \times B$ transport strongly in the low collisional case but is less effective in the high collisional case. Basic assumptions of the simulations discussed in Refs. 18 and 19, such as the electrostatic approximation, the neglect of sound waves, and the strong impact on the resonant large scale dynamics, giving reason for a strong reduction in the number of modes taken into account, could be verified in our calculation for the high collisional case. But the results obtained with the RBM3D code, namely, the decrease of density fluctuations and an almost unchanged total (static+turbulent) radial $E \times B$ transport could not be recovered. As shown by means of energy balances the reason for this discrepancy can be found in a density dissipation of the RBM3D model, which is increased in the presence of externally induced magnetic perturbation fields. According to the fact that the RBM3D model is a subset of the model considered in this work, this effect is also present in our simulations, but it is compensated due to the implementation of the full drift-Alfvén dynamics. All things considered the numerical results of our turbulence studies demonstrate that a low collisionality makes the plasma more susceptible for an enhancement of turbulent transport due to magnetic perturbation fields. The turbulent transport is increased strongly for the less collisional, electromagnetic drift-Alfvén turbulence-dominated plasma, whereas in the more collisional plasma with strong electrostatic ballooning turbulence the turbulent transport is almost unchanged. But due to a strong static contribution an overall increase of cross field transport is found in both regimes. To make a statement about how these effects can alter the plasma transport in real experiments a more sophisticated modeling is required, taking into account warm ions and temperature dynamics. This is planned for the near future.

\section{APPENDIX: ENERGY THEOREM FOR THE FOUR-FIELD MODEL}

For the purpose of numerical diagnosis and analysis of the dynamics of our model system [Eqs. (1)-(4)] we consider the fluctuation free-energy density functional which in the DALF3 model represents self-consistency in the energy conservation and transfer. (See Fig. 18.) Here, we add the effects 


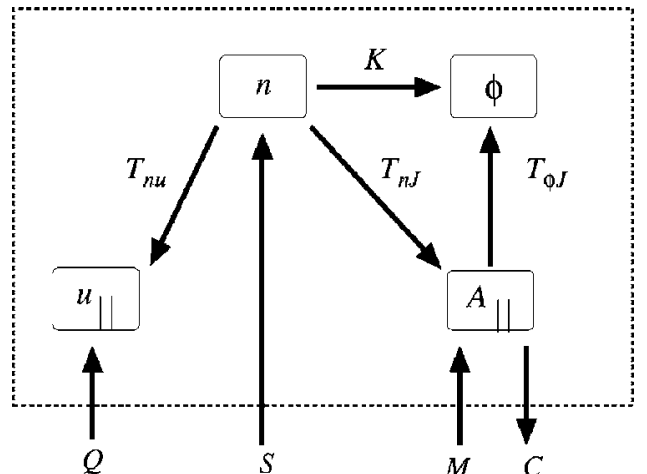

FIG. 18. Schematic of picture energy flow in the model system Eqs. (1)-(4). The direction of the arrows corresponds to a positive value of the respective quantity defined by (A11)-(A18). The dashed line indicates the boundary of the open model system.

arising from the external magnetic-field perturbation. The free-energy density is given by

$$
\widetilde{U}=\frac{1}{2} n M_{i}\left(\widetilde{v}_{E}^{2}+\widetilde{v}_{\|}^{2}\right)+\frac{\widetilde{B}^{2}}{2 \mu_{0}}+\frac{m_{e}}{2 e^{2} n} \widetilde{J}_{\|}^{2}+\frac{1}{2} n T_{e} \frac{\widetilde{n}^{2}}{n^{2}},
$$

where $\widetilde{v}_{E}^{2}=\left|\boldsymbol{\nabla}_{\perp} \widetilde{\phi}\right|^{2} / B^{2}$ and $\widetilde{B}^{2}=\left|\boldsymbol{\nabla}_{\perp} \widetilde{A}_{\|}\right|^{2}$. The time derivative of the total free energy $\widetilde{E}=\langle\widetilde{U}\rangle_{V}$ is then

$$
\frac{\partial \widetilde{E}}{\partial t}=\frac{\partial \widetilde{E}_{\phi}}{\partial t}+\frac{\partial \widetilde{E}_{u_{\|}}}{\partial t}+\frac{\partial \widetilde{E}_{n}}{\partial t}+\frac{\partial \widetilde{E}_{A_{\|}}}{\partial t},
$$

where

$$
\begin{aligned}
& \frac{\partial \widetilde{E}_{\phi}}{\partial t}=-\left\langle\frac{n M_{i}}{B^{2}} \widetilde{\phi} \frac{\partial \nabla_{\perp}^{2} \widetilde{\phi}}{\partial t}\right\rangle_{V}, \\
& \frac{\partial \widetilde{E}_{u_{\|}}}{\partial t}=\left\langle n M_{i} \widetilde{u}_{\|} \frac{\partial \widetilde{u}_{\|}}{\partial t}\right\rangle_{V}, \\
& \frac{\partial \widetilde{E}_{n}}{\partial t}=\left\langle\frac{T_{e}}{n} \widetilde{n} \frac{\partial \widetilde{n}}{\partial t}\right\rangle_{V}, \\
& \frac{\partial \widetilde{E}_{A_{\|}}}{\partial t}=\left\langle\widetilde{J}_{\|}\left(\frac{\partial \tilde{A}_{\|}}{\partial t}+\frac{m_{e}}{e^{2} n} \frac{\partial \widetilde{J}_{\|}}{\partial t}\right)\right\rangle_{V} .
\end{aligned}
$$

The subscripted angle brackets $\langle\cdots\rangle_{V}$ denote the volume average. Inserting Eqs. (1)-(4) into each corresponding piece of this expression leads to (we neglect surface terms)

$$
\begin{aligned}
& \frac{\partial \widetilde{E}_{\phi}}{\partial t}=T_{\phi J}+K, \\
& \frac{\partial \widetilde{E}_{u_{\|}}}{\partial t}=Q+T_{n u}, \\
& \frac{\partial \widetilde{E}_{n}}{\partial t}=S-T_{n J}-T_{n u}-K,
\end{aligned}
$$

$$
\frac{\partial \widetilde{E}_{A_{\|}}}{\partial t}=M+T_{n J}-T_{\phi J}-C,
$$

where the different contributions are defined as

$$
\begin{aligned}
& S=-\left\langle\frac{T_{e}}{n} \tilde{n} \widetilde{\mathbf{v}}_{E} \cdot \nabla_{n}\right\rangle_{V}, \\
& M=\left\langle\frac{T_{e}}{e n} \widetilde{J}_{\|} \nabla_{\|}\right\rangle_{V}, \\
& Q=-\left\langle T_{e} \widetilde{u}_{\|} \nabla_{\|}\right\rangle_{V}, \\
& T_{n J}=\left\langle\frac{T_{e}}{n e} \widetilde{J}_{\|} \nabla_{\|} \tilde{n}\right\rangle_{V}, \\
& T_{n u}=-\left\langle T_{e} \widetilde{u}_{\|} \nabla_{\|} \tilde{n}\right\rangle_{V}, \\
& T_{\phi J}=\left\langle\widetilde{J}_{\|} \nabla_{\|} \widetilde{\phi}\right\rangle_{V}, \\
& K=-\left\langle T_{e} \widetilde{n} \mathcal{K}(\widetilde{\phi})\right\rangle_{V}, \\
& C=\left\langle\eta_{\|} \widetilde{J}_{\|}^{2}\right\rangle_{V} .
\end{aligned}
$$

The quantities $S, M$, and $Q$ act as sources for the turbulent dynamics, extracting energy out of the background density gradient. The quantity $C$ is a sink for the fluctuation free energy due to resistivity, and $T_{n J_{\|}}, T_{n u_{\|}}$, and $T_{\phi J_{\|}}$are transfer terms due to drift-Alfvén-wave and sound wave dynamics. Also $K$ is a transfer term, which represents the ballooning mechanism (toroidal compression of diamagnetic flows and currents and of the $E \times B$ velocity). It is obvious that the transfer terms do not contribute to the temporal change of the total free energy in the volume considered and it follows that

$$
\frac{\partial \tilde{E}}{\partial t}=S+M+Q-C
$$

However, the transfer pieces in the energy theorem are well suited to gain insight into the interaction of the various dynamical mechanisms in the model system. For the purpose of analyzing the importance of the different contributions in the computational results we also define the following growth rates:

$$
\begin{aligned}
& \nu_{S}=\frac{S}{\widetilde{E}}, \quad \nu_{M}=\frac{M}{\widetilde{E}}, \quad \nu_{Q}=\frac{Q}{\widetilde{E}}, \quad \nu_{C}=\frac{C}{\widetilde{E}}, \\
& \nu_{n J}=\frac{T_{n J}}{\widetilde{E}}, \quad \nu_{n u}=\frac{T_{n u}}{\widetilde{E}}, \quad \nu_{\phi J}=\frac{T_{\phi J}}{\widetilde{E}}, \quad \nu_{K}=\frac{K}{\widetilde{E}},
\end{aligned}
$$

and according to Eqs. (A7)-(A10) we define the growth rates for the different contributions to the total energy,

$$
\begin{aligned}
& \nu_{\phi}=\nu_{\phi J}+\nu_{K}, \quad \nu_{u_{\|}}=\nu_{Q}+\nu_{n u}, \\
& \nu_{n}=\nu_{S}-\nu_{n J}-\nu_{n u}-\nu_{K},
\end{aligned}
$$




$$
\nu_{A_{\|}}=\nu_{M}+\nu_{n J}-\nu_{\phi J}-\nu_{C},
$$

such that

$$
\frac{\partial \ln \tilde{E}}{\partial t}=\nu_{\phi}+\nu_{u_{\|}}+\nu_{n}+\nu_{A_{\|}}=\nu_{S}+\nu_{M}+\nu_{Q}-\nu_{C} .
$$

${ }^{1}$ G. A. Massel and M. R. Feix, Phys. Fluids 11, 2738 (1968).

${ }^{2}$ A. B. Rechester and M. N. Rosenbluth, Phys. Rev. Lett. 40, 38 (1978).

${ }^{3}$ T. H. Stix, Nucl. Fusion 18, 353 (1978).

${ }^{4}$ H. E. Mynick and J. A. Krommes, Phys. Fluids 23, 1229 (1980).

${ }^{5}$ J. A. Krommes, C. Oberman, and R. G. Kleva, J. Plasma Phys. 30, 11 (1983).

${ }^{6}$ A. J. Wootton, H. Tsui, and S. Prager, Plasma Phys. Controlled Fusion 34, 2023 (1992).

${ }^{7}$ M. R. Stoneking, S. A. Hokin, S. C. Prager et al., Phys. Rev. Lett. 73, 549 (1994).

${ }^{8}$ R. J. Bickerton, Plasma Phys. Controlled Fusion 39, 339 (1997).

${ }^{9}$ A. Grosman, T. E. Evans, Ph. Ghendrih et al., J. Nucl. Mater. 176-177, 493 (1990).

${ }^{10}$ A. Grosman, Ph. Ghendrih, C. de Michelis et al., J. Nucl. Mater. 196-198, 59 (1992).

${ }^{11} \mathrm{Ph}$. Ghendrih, A. Grosman, and H. Capes, Plasma Phys. Controlled Fusion 38, 1653 (1996).

${ }^{12}$ K. H. Finken, S. S. Abdullaev, A. Kaleck, and G. H. Wolf, Nucl. Fusion 39, 637 (1999).

${ }^{13}$ S. S. Abdullaev, K. H. Finken, M. W. Jakubowski, S. V. Kasilov, M.
Kobayashi, D. Reiser, M. A. Runov, and R. Wolf, Nucl. Fusion 43, 299 (2003).

${ }^{14}$ Th. Eich, D. Reiser, and K. H. Finken, J. Nucl. Mater. 290-293, 849 (2001).

${ }^{15}$ M. Z. Tokar', Phys. Plasmas 6, 2808 (1999).

${ }^{16}$ H. Capes, A. Samain, Ph. Ghendrih, and F. Nguyen, Contrib. Plasma Phys. 32, 192 (1992).

${ }^{17}$ A. Samain, H. Capes, Ph. Ghendrih, and F. Nguyen, Phys. Fluids B 5, 471 (1993).

${ }^{18}$ P. Beyer, X. Garbet, and P. Ghendrih, Phys. Plasmas 5, 4271 (1998).

${ }^{19}$ P. Beyer, X. Garbet, S. Benkadda, P. Ghendrih, and Y. Sarazin, Plasma Phys. Controlled Fusion 44, 2167 (2002).

${ }^{20}$ B. Scott, Phys. Plasmas 8, 447 (2001).

${ }^{21}$ B. D. Scott, New J. Phys. 4, 52 (2002)

${ }^{22}$ B. D. Scott, Plasma Phys. Controlled Fusion 39, 1635 (1997)

${ }^{23}$ M. Wakatani and A. Hasegawa, Phys. Fluids 27, 611 (1984).

${ }^{24}$ B. D. Scott, Phys. Fluids B 4, 2468 (1992).

${ }^{25}$ F. L. Hinton and C. W. Horton, Jr., Phys. Fluids 14, 116 (1971).

${ }^{26}$ S. I. Braginskii, Rev. Plasma Phys. 1, 205 (1965).

${ }^{27}$ A. Kendl, B. D. Scott, R. Ball, and R. L. Dewar, Phys. Plasmas 10, 3684 (2003).

${ }^{28}$ B. Scott, A. B. Hassam, and J. F. Drake, Phys. Fluids 28, 275 (1985).

${ }^{29}$ R. E. Waltz, Phys. Fluids 28, 577 (1985).

${ }^{30}$ S. J. Camargo, B. Scott, and D. Biskamp, Phys. Plasmas 3, 3912 (1996).

${ }^{31}$ M. A. Beer, S. C. Cowley, and C. W. Hammett, Phys. Plasmas 2, 2687 (1995).

${ }^{32}$ B. Scott, Phys. Plasmas 5, 2334 (1998).

${ }^{33}$ J. Wesson, Tokamaks, 2nd ed. (Clarendon, Oxford, 1997), p. 369.

${ }^{34}$ B. Scott, Plasma Phys. Controlled Fusion 45, A385 (2003). 